\title{
Star formation and gas flows in the centre of the NUGA galaxy NGC 1808 observed with SINFONI*
}

\author{
Gerold Busch $^{1}$, Andreas Eckart ${ }^{1,2}$, Mónica Valencia-S. ${ }^{1}$, Nastaran Fazeli ${ }^{1}$, Julia Scharwächter ${ }^{3}$, \\ Françoise Combes ${ }^{4}$, and Santiago García-Burillo ${ }^{5}$ \\ 1 I. Physikalisches Institut der Universität zu Köln, Zülpicher Str. 77, 50937 Köln, Germany
e-mail: [busch; eckart] @ph1. uni-koeln. de
2 Max-Planck-Institut für Radioastronomie, Auf dem Hügel 69, 53121 Bonn, Germany
3 Gemini Observatory, Northern Operations Center, 670 N. A’ohoku Place, Hilo, HI, 96720, USA
4 LERMA, Observatoire de Paris, College de France, PSL, CNRS, Sorbonne Univ., UPMC, 75014 Paris, France
5 Observatorio Astronómico Nacional (OAN) - Observatorio de Madrid, Alfonso XII 3, 28014 Madrid, Spain
}

Received 30 July 2016 / Accepted 22 November 2016

\section{ABSTRACT}

\begin{abstract}
NGC 1808 is a nearby barred spiral galaxy which hosts young stellar clusters in a patchy circumnuclear ring with a radius of $\sim 240 \mathrm{pc}$. In order to study the gaseous and stellar kinematics and the star formation properties of the clusters, we perform seeing-limited $H+K$ band near-infrared integral-field spectroscopy with SINFONI of the inner $\sim 600 \mathrm{pc}$. From the $M_{\mathrm{BH}}-\sigma_{*}$ relation, we find a black hole mass of a few $10^{7} M_{\odot}$. We estimate the age of the young stellar clusters in the circumnuclear ring to be $\lesssim 10$ Myr. No age gradient along the ring is visible. However, the starburst age is comparable to the travel time along the ring, indicating that the clusters almost completed a full orbit along the ring during their lifetime. In the central $\sim 600 \mathrm{pc}$, we find a hot molecular gas mass of $\sim 730 M_{\odot}$ which, with standard conversion factors, corresponds to a large cold molecular gas reservoir of several $10^{8} M_{\odot}$, in agreement with $\mathrm{CO}$ measurements from the literature. The gaseous and stellar kinematics show several deviations from pure disc motion, including a circumnuclear disc and signs of a nuclear bar potential. In addition, we confirm streaming motions on the $\sim 200 \mathrm{pc}$ scale that have recently been detected in $\mathrm{CO}(1-0)$ emission. Thanks to the enhanced angular resolution of $<1^{\prime \prime}$, we find further streaming motion within the inner arcsecond that had not been detected until now. Despite the flow of gas towards the centre, no signs of significant AGN activity are found. This raises the question: will the infalling gas fuel an AGN or star formation?
\end{abstract}

Key words. galaxies: active - galaxies: starburst - galaxies: nuclei - galaxies: individual: NGC 1808 - infrared: galaxies galaxies: kinematics and dynamics -

\section{Introduction}

It is now well established that galaxies, at least those with massive spheroidal components, harbour supermassive black holes (SMBH) in their nuclei (e.g. Kormendy \& Richstone 1995; Kormendy \& Ho 2013). Active galactic nuclei (AGNs) are fuelled by accretion onto their central SMBHs. Tight relations between the mass of the SMBH and the properties of the host galaxy (mainly its spheroidal component) are interpreted as a sign of a common evolution of SMBH and host galaxy (Ferrarese \& Merritt 2000; Gebhardt et al. 2000; Marconi \& Hunt 2003; Häring \& Rix 2004; Graham \& Driver 2007; Gültekin et al. 2009; Graham 2012; Läsker et al. 2014; Savorgnan et al. 2016; Savorgnan 2016; Graham 2016). Therefore, a full understanding of how AGNs are fuelled and what prevents other galaxies from being fuelled (and thereby being quiescent instead of active) is crucial to understanding the evolution of galaxies from high redshift to the local Universe.

In high-luminosity AGNs the onset of nuclear activity is linked to large-scale (kpc) perturbations like bars and galaxy interactions which can trigger a gas inflow

\footnotetext{
$\star$ Based on observations with ESO-VLT, STS-Cologne GTO proposal ID 094.B-0009(A) and ESO archival data, proposal nos 074.A-9011(A) and 075.B-0648(A).
}

(e.g. Sanders et al. 1988; Hopkins \& Quataert 2010; Hilz et al. 2013), while low-luminosity AGNs seem to be dominated by secular evolution (e.g. Hopkins et al. 2008; Hopkins \& Quataert 2010; Kormendy et al. 2011). Possible feeding mechanisms are bars, secondary/nuclear bars, $m=1$ instabilites, warps, nuclear spirals, and stellar winds (García-Burillo et al. 2005; GarcíaBurillo \& Combes 2012, and references therein), and they cover a wide range of scales from host galaxy $(\gtrsim 1 \mathrm{kpc})$ to nuclear scales $(\lesssim \mathrm{pc})$. A further difficulty is caused by the different timescales of star formation and AGN activity, in particular the fact that even during a total AGN duty cycle of $10^{8} \mathrm{yr}$, the AGN might "flicker" on much lower timescales of $10^{5} \mathrm{yr}$ (e.g. Hickox et al. 2014; Schawinski et al. 2015).

The NUclei of GAlaxies (NUGA) survey was established to systematically investigate the issue of nuclear fuelling for nearby galaxies. NUGA started off as an IRAM key project (PIs: Santiago García-Burillo and Françoise Combes; see García-Burillo et al. 2003) on the northern hemisphere and is continuing now on the southern hemisphere with the Atacama Large Millimeter/submillimeter Array (ALMA) as the millimetric investigations can prospectively be performed with a superior angular resolution and sensitivity (Combes et al. 2013, 2014; García-Burillo et al. 2014). NUGA comprises a sample of 30 nearby AGNs covering all stages of nuclear activity 
(Seyferts - LINERs - starbursts). The combined data sets allow a first systematic study of gas kinematics covering scales from a few tens of parsec to the outer few tens of kiloparsec.

Complementary integral-field spectroscopy (IFS) data are taken in the near-infrared (see e.g. the cases of NGC 1433 and NGC 1566; Smajić et al. 2014, 2015). With the near-infrared data we can include information on the hot molecular and atomic gas and their excitation mechanisms (e.g. Zuther et al. 2007; Mazzalay et al. 2013; Smajić et al. 2014), and on the properties of the central engine (in particular black hole mass and hidden broad line region; see the case of NGC 7172 in Smajić et al. 2012). Furthermore IFS in the near-infrared is the ideal tool for studying stellar populations and star formation in (dust-obscured) centres of galaxies (e.g. Böker et al. 2008; Riffel et al. 2009; Bedregal et al. 2009; Valencia-S. et al. 2012; Falcón-Barroso et al. 2014; Busch et al. 2015; Smajić et al. 2015). IFS also allows kinematically decoupled regions like nuclear discs and spatially resolving inflows and outflows to be found in many galaxies (e.g. Riffel et al. 2008; Storchi-Bergmann et al. 2010; Müller-Sánchez et al. 2011; Scharwächter et al. 2013; Davies et al. 2014a; Riffel et al. 2015b; Diniz et al. 2015).

In this paper, we present near-infrared IFS of the galaxy NGC 1808 from the NUGA sample which is a (R)SAB(s)a barred spiral galaxy (de Vaucouleurs et al. 1991). NGC 1808 has been early reported to contain "hotspots" near the galaxy nucleus (Morgan 1958; Sérsic \& Pastoriza 1965; Pastoriza 1975). These hotspots are mainly prominent in radio and/or near- and mid-infrared emission and trace young stellar clusters and their associated supernova remnants (Forbes et al. 1992; Collison et al. 1994; Krabbe et al. 1994; Kotilainen et al. 1996; Tacconi-Garman et al. 1996; Galliano et al. 2005; Galliano \& Alloin 2008). The near-infrared spots are located in a circumnuclear ring with radius $r \sim 280$ pc (Comerón et al. 2010). Some authors have reported that the galaxy contains a weak AGN (following the classification of Veron-Cetty \& Veron 1985); however, others disagree (e.g. Forbes et al. 1992; Phillips 1993; Krabbe et al. 2001; Dopita et al. 2015). We discuss the possible presence of an AGN in Sect. 4.6.

Prominent dust lanes on kiloparsec scales (pointing from the nucleus in the north-east direction) that are visible in optical images and peculiar motions of $\mathrm{H} \mathrm{I}$ are interpreted as indications of an outflow of neutral and ionised gas (Phillips 1993; Koribalski et al. 1993). A possible tidal interaction with NGC 1792 has been discussed (Dahlem et al. 1990; Koribalski et al. 1993).

NGC 1808 has recently been mapped in ${ }^{12} \mathrm{CO}(J=1-0)$ by Salak et al. (2016) at a resolution of $2^{\prime \prime}(\sim 100 \mathrm{pc})$. In the centre they find a compact circumnuclear disc $(r<200 \mathrm{pc})$ and a molecular gas ring with radius $r \sim 500 \mathrm{pc}$. By analysing the gas kinematics, they find several components of non-circular motion: a spiral pattern in the inner disc $(r<400 \mathrm{pc})$ and gas streaming motion on the inner side of one spiral arm, as well as a molecular gas outflow from the nuclear starburst region $(r<250 \mathrm{pc})$ which is spatially coincident with one of the mentioned dust lanes.

This paper presents a first comprehensive near-infrared IFS study of the central kpc of NGC 1808 . In comparison to previous studies of the emission line distribution, our data has higher spatial resolution $\left(\lesssim 1^{\prime \prime}\right)$ and much higher sensitivity so that we can trace the morphology in great detail. The combination of a high spectral coverage $(1.45-2.45 \mu \mathrm{m}$ for the $H+K$-band grating) and high spectral resolution ( $R=4000$ for the $K$-band grating) allows us to get detailed maps of stellar and gaseous kinematics and to study diagnostic line ratios in a spatially resolved way.

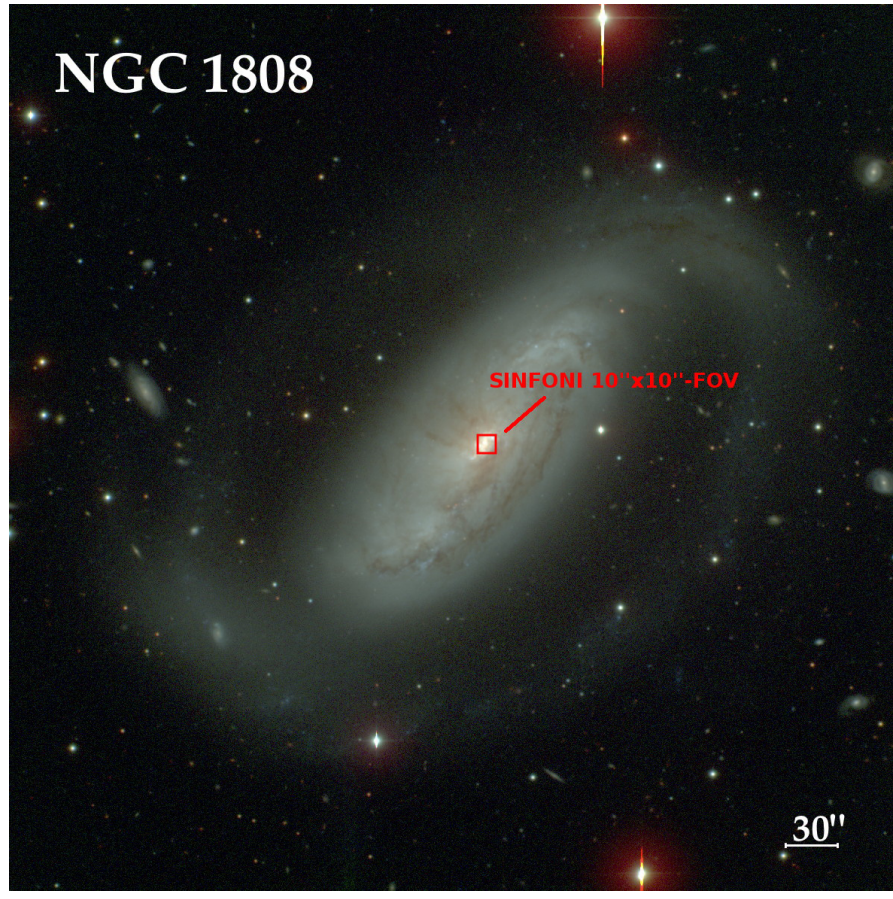

Fig. 1. Optical image of the barred spiral galaxy NGC 1808. The $10^{\prime \prime} \times$ $10^{\prime \prime}$ field of view of SINFONI is indicated. Image courtesy: CarnegieIrvine Galaxy Survey (Ho et al. 2011).

Only with instruments like SINFONI can we study combined kinematical information and excitation mechanisms from a single, homogeneous data set.

Figure 1 shows an optical image of NGC 1808, and indicates the field of view (FOV) of the SINFONI near-infrared IFS data analysed in detail in this paper. Figure 3 shows a HST falsecolour image of the central region.

The paper is structured as follows. In Sect. 2 we present the observations used for this analysis. In Sect. 3 we present the results of the analysis of the SINFONI data cubes (in particular emission line maps, as well as stellar and gaseous kinematics), which we then discuss with regard to previous results from the literature in Sect. 4. Section 5 gives a short summary and conclusions from this work.

In order to calculate spatial scales and luminosities, we adopt a luminosity distance of $D_{L}=(12.8 \pm 1.2) \mathrm{Mpc}$ (Tully et al. 2009), which corresponds to a scale of $62 \mathrm{pc} \mathrm{arcsec}^{-1}$.

\section{Observations and data reduction}

\subsection{SINFONI near-infrared integral-field spectroscopy}

The analysis of NGC 1808 in this paper is based on nearinfrared IFS data obtained with SINFONI (Eisenhauer et al. 2003; Bonnet et al. 2004) at the Very Large Telescope (VLT) of the European Southern Observatory (ESO) in Chile.

We observed the central region of NGC 1808 in seeinglimited mode on October 6, 2014. The FOV of single exposures in this mode is $8^{\prime \prime} \times 8^{\prime \prime}$, which results in a spatial sampling of 0.125 arcsec pixel $^{-1}$. We used a jitter pattern with offsets of $\pm 1^{\prime \prime}$ to minimise the effect of bad pixels and increase the FOV of the combined cube to $10^{\prime \prime} \times 10^{\prime \prime}$ (which corresponds to a linear scale of $620 \mathrm{pc})$. We spent $1500 \mathrm{~s}(10 \times 150 \mathrm{~s})$ on-source time using the $H+K$ grating with a spectral resolution of $R \approx 1500$. This grating has a wide simultaneous wavelength coverage which is useful for analysis of emission line ratios. Furthermore, we spent 


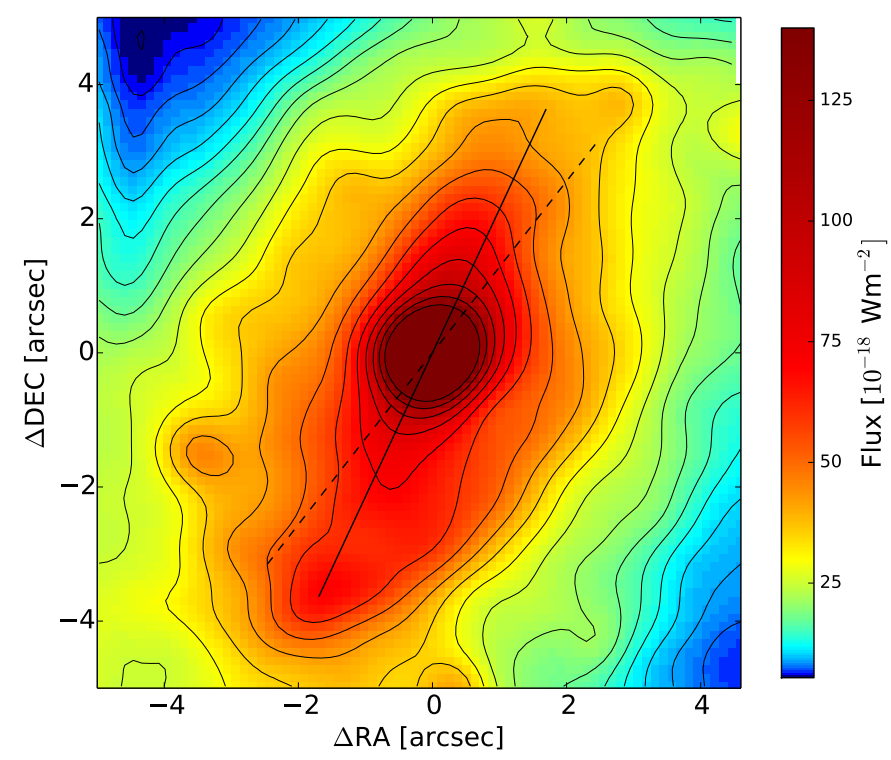

Fig. 2. $K$-band continuum image extracted from the SINFONI data cube. The solid line marks the PA of the nuclear bar $\left(\sim 115^{\circ}\right.$, measured from north to east), while the dashed line marks the line of nodes of the stellar kinematics (Sect. 4.1).

3000 s $(20 \times 150$ s $)$ of on-source time using the $K$-band grating, which results in a higher spectral resolution of $R \approx 4000$ and is ideal for analysing the stellar dynamics.

Furthermore, we use seeing-limited (FOV: $8^{\prime \prime} \times 8^{\prime \prime}$ ) SINFONI $J$-band data from the ESO-archive (proposal nos. 074.A9011(A) and 075.B-0648(A)) that have a total on-source exposure time of $1200 \mathrm{~s}$. These data are needed to determine the extinction by using the line ratio between the hydrogen emission lines $\mathrm{Br} \gamma$ in the $K$ band and $\mathrm{Pa} \beta$ in the $J$ band.

The pipeline which is delivered by ESO was used for data reduction up to single-exposure cube reconstruction. For alignment, final coaddition, and telluric correction, we use our own PYTHON and IDL routines. For a more detailed description of the reduction and calibration, we refer the reader to Busch et al. (2015) and Smajić et al. (2014). Figure 2 shows a $K$-band continuum image that was extracted from the SINFONI data cube.

\subsection{Hubble Space Telescope imaging data}

We retrieved an image of the central region of NGC 1808 from the Hubble Legacy Archive ${ }^{1}$. The images were taken with the Wide Field and Planety Camera 2 of the Hubble Space Telescope in the $F 814 W, F 675 W$, and $F 658 N$ filters. The observations took place in August 1998 (proposal ID 6872, PI: James Flood). In Fig. 3 we show an RGB composition of these images (red: $F 814 W$, green: $F 675 W$, blue: F658N) and indicate the apertures of the spots that we analyse in detail.

\section{Results}

\subsection{Emission line flux distributions}

The near-infrared emission lines trace the hot molecular and ionised atomic gas phase. This gas is predominantly associated with the sites of young star formation (H II regions, shocks, hot surfaces of molecular clouds) or non-thermal nuclear activity (nuclear ionisation, winds, and shock regions).

\footnotetext{
1 http://hla.stsci.edu/
}

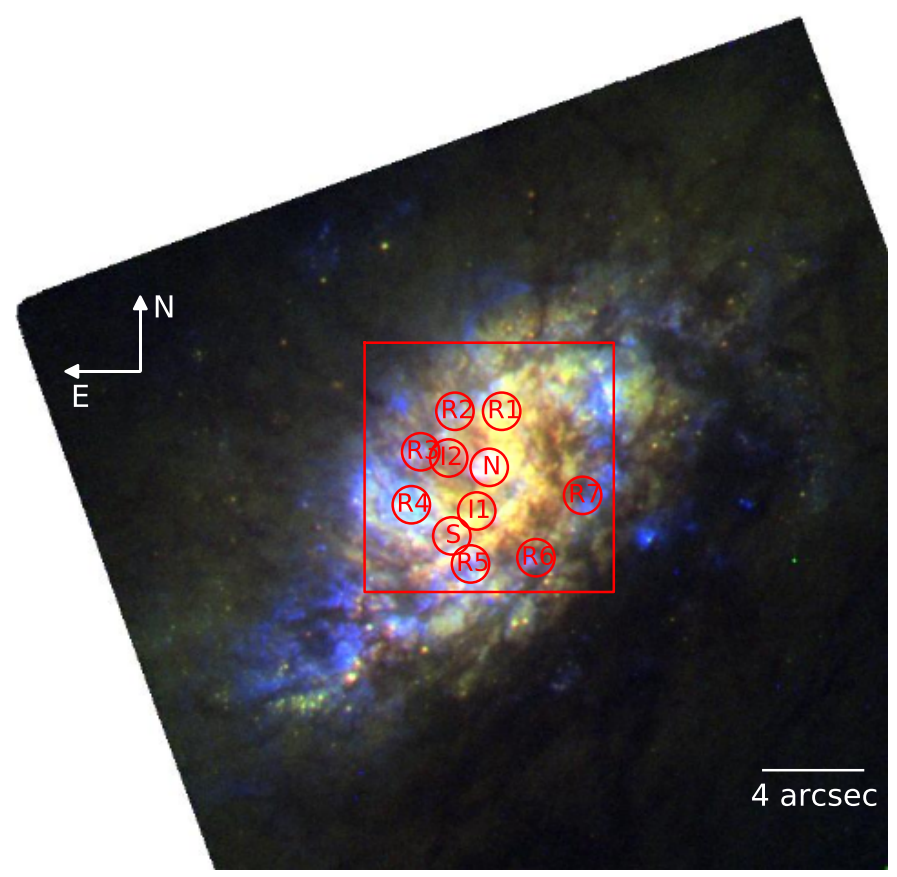

Fig. 3. HST false-colour image of the central region of NGC 1808 (red: $F 814 \mathrm{~W}$, green: $F 675 \mathrm{~W}$, blue: $F 658 N$ ). The apertures that are analysed in detail are indicated by red circles.

The data cubes show a wide variety of emission lines, including several hydrogen recombination lines (which trace fully ionised regions), molecular hydrogen lines, and the shock tracer [Fe II] (which traces partially ionised regions), as well as stellar absorption lines.

We use the PYTHON implementation of MPFITEXPR, which is based on the Levenberg-Marquardt algorithm (Markwardt 2009; Moré 1978), to generate maps of the emission line flux distributions. The line fluxes were determined by fitting a Gaussian function to the line profile. The continuum was subtracted by fitting a linear function to the continuum emission in two spectral windows, to the left and right of the emission line. The emission line maps are clipped to have uncertainties of less than $30 \%$.

In Fig. 4, we show the maps of $\mathrm{Pa} \alpha, \mathrm{H}_{2} \lambda 2.12 \mu \mathrm{m}$, and the $H$-band emission line [Fe II] at $1.644 \mu \mathrm{m}$, which we analyse in the following.

All three mapped gas types ( $\mathrm{Pa} \alpha, \mathrm{H}_{2} \lambda 2.122 \mu \mathrm{m},[\mathrm{Fe}$ II]) have their absolute peaks in the centre, coinciding with the peak of the continuum flux, which is marked by a cross. Apart from this they show quite different flux distributions.

Ionised gas as traced by the hydrogen recombination lines $\mathrm{Pa} \alpha$ and $\mathrm{Br} \gamma$ is located in a ring around the centre which has a radius of $\sim 4^{\prime \prime}(\approx 240 \mathrm{pc})^{2}$. With our high spatial resolution data, we confirm the asymmetry mentioned by Krabbe et al. (1994) and Kotilainen et al. (1996), namely that the patches to the south-east show higher flux levels compared to the west side. Furthermore, there seem to be several gaps in the ring, the largest in the northwest, that give the ring an overall patchy structure. We note that in the region between ring and nucleus $\mathrm{Pa} \alpha$ and $\mathrm{Br} \gamma$ are also detected, but at a lower intensity than in the ring. A tail-like structure in the south-east is visible in the maps of (Kotilainen et al. 1996, which have a FOV of $20^{\prime \prime} \times 20^{\prime \prime}$ but lower angular

\footnotetext{
2 This circumnuclear ring is not the same as the $500 \mathrm{pc}$ molecular gas ring that Salak et al. (2016) find in their recent CO data. Our FOV is limited to the region that they call "circumnuclear disk".
} 

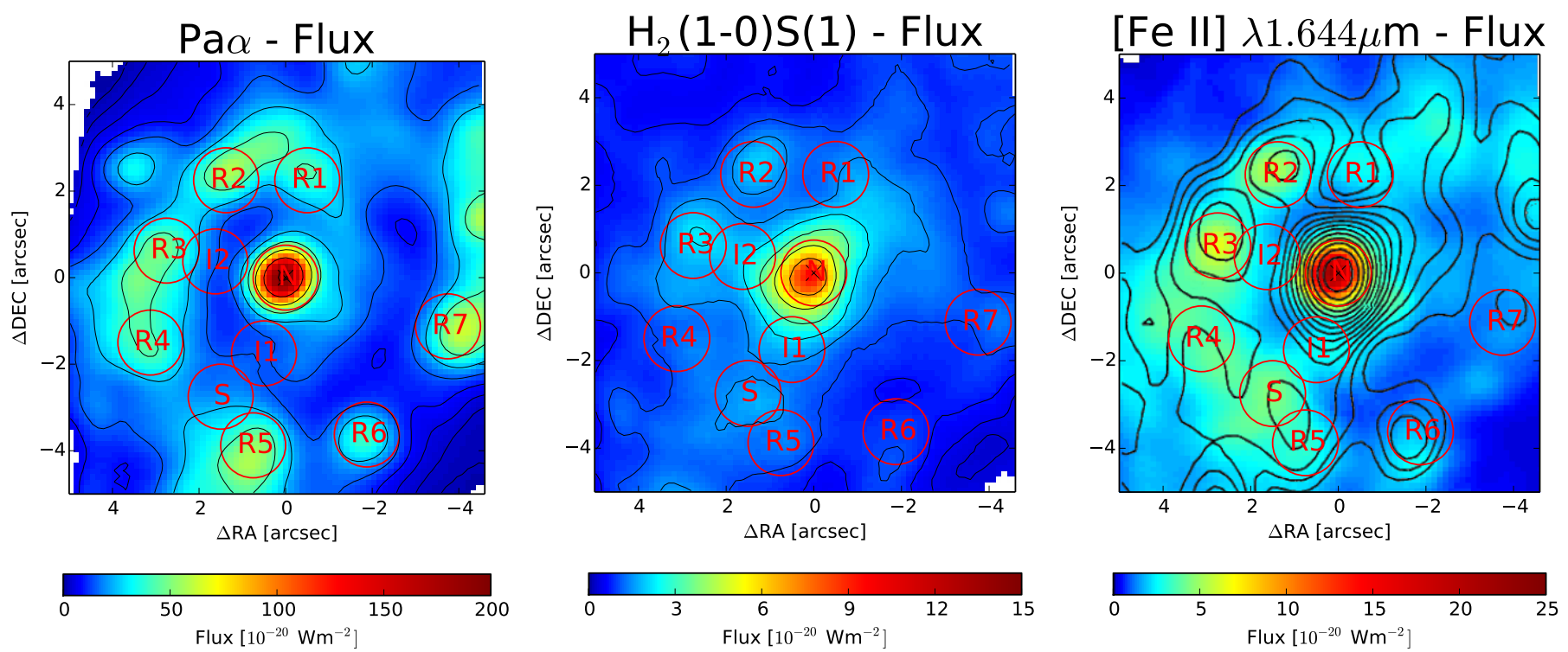

Fig. 4. Flux maps of the $\mathrm{Pa} \alpha, \mathrm{H}_{2} \lambda 2.12 \mu \mathrm{m}$, and [Fe II] $\lambda 1.64 \mu \mathrm{m}$ emission lines. The [Fe II] map is overlaid with the $3.6 \mathrm{~cm}$ radio-continuum image of Collison et al. (1994) as printed in Kotilainen et al. (1996). The molecular hydrogen shows a more centrally concentrated distribution; this is different from the distribution of ionised hydrogen and the shock tracer [Fe II] which show emission in the centre, but also in a circumnuclear ring with radius $4^{\prime \prime} \approx 240 \mathrm{pc}$.

resolution) and is probably part of the gas spiral arms that can often be seen on kpc scales (see e.g. Busch et al. 2015).

In the emission of hot molecular hydrogen (traced by the $\mathrm{H}_{2} \lambda 2.12 \mu \mathrm{m}$ emission line) the circumnuclear ring, found in $\mathrm{Pa} \alpha$ and $\mathrm{Br} \gamma$, is less prominent. Some of the patches in the ring also show an enhanced flux level in $\mathrm{H}_{2}$, but the contrast between the ring and the inter-ring region is much lower in $\mathrm{H}_{2}$ than in $\mathrm{Pa} \alpha$. Furthermore, we find that the flux in the centre is more extended than in $\mathrm{Pa} \alpha$. To quantify this finding, we fit a Gaussian to the central peak. For $\mathrm{H}_{2}$, the FWHM is $1^{\prime \prime} ! 3$, but only 0 '!9 for $\mathrm{Pa} \alpha$. Furthermore, we note that we find strong $\mathrm{H}_{2}$ flux between the ring and the nucleus in the eastern direction (labelled "I2" in Fig. 4).

At first glance, the flux distribution of [Fe II] looks very similar to that of $\mathrm{Pa} \alpha$ and $\mathrm{Br} \gamma$, i.e. it also shows a prominent circumnuclear ring. However, two differences are striking. First, some $\mathrm{Pa} \alpha$ patches show a much lower flux level in [Fe II] than the others. We use this to estimate starburst ages. Second, the [Fe II] shows strong emission in the south-east side of the ring (marked with "S" in Fig. 4) that does not coincide with a $\mathrm{Pa} \alpha$ and $\mathrm{Br} \gamma$ peak. We analyse this region in more detail later.

In order to analyse the excitation properties, star formation, and more in the nucleus (N), ring (R1 to R7), and in between (I1, I2, and S), we chose eleven apertures that are marked in Figs. 3 and 4. We extract spectra from circular apertures with radii $r=0,775$. The spectra are shown in Fig. 5.

Before we measure the emission lines, we perform a subtraction of the stellar continuum with the PYTHON implementation of the Penalized Pixel-Fitting method (pPXF, Cappellari $\&$ Emsellem 2004). We use a set of synthetic model spectra by Lançon et al. (2007) with solar abundances, an effective temperature range $T_{\text {eff }}=2900-5900 \mathrm{~K}$, gravities of $\log \left(\mathrm{g} / \mathrm{cm} \mathrm{s}^{-2}\right)=$ $[-1.0,-0.5,0.0,+1.0]$, and masses $1 M_{\odot}$ and $15 M_{\odot}$. The modelled stellar continuum is shown in Fig. 5 in red. We then subtract these models from the original spectra and use the residual for the emission line fits.

For the emission line fits, we use the PYTHON implementation of MPFITEXPR which is based on the Levenberg-Marquardt algorithm (Markwardt 2009; Moré 1978). All emission lines are fitted with Gaussian functions. In order to estimate the uncertainties of the parameters, we perform a Monte Carlo simulation with 100 iterations. In each iteration Gaussian noise is added to the input spectrum, with the width corresponding to the root mean square of the residual spectrum (input spectrum - fit). We then take the mean of the 100 fit results as the best fit and the standard deviations as the uncertainties of the fit parameter (see also Busch et al. 2016). The measured line fluxes are presented in Tables 1 and 2.

\subsection{Emission line ratios}

In the following section, we calculate and map emission line ratios. Emission line ratios allow us to trace the extinction and the UV radiation field of the ISM as well as possible shocks.

\subsubsection{Extinction correction}

Especially in the case of AGNs and starburst galaxies, reddening induced by dust can significantly affect the measured emission line fluxes. Near-infrared observations have the advantage that this effect is much less prominent than in the optical (extinction is lower by approximately a factor of 10 in magnitude). Therefore observations, e.g. of the Galactic Centre or dust enshrouded galactic nuclei (see e.g. Smajić et al. 2012), are often only feasible when going from optical to longer near-infrared wavelengths. However, even in the near-infrared, extinction effects can still be significant and should therefore be corrected.

Emission line fluxes can be corrected following the relation $F_{\text {intr }}=F_{\text {obs }} \times 10^{0.4 \times A(\lambda)}$ between observed flux $F_{\text {obs }}$ and intrinsic flux $F_{\text {intr. }}$. We use the extinction law by Calzetti et al. (2000) to get an expression for the extinction at wavelength $\lambda$ (in $\mu \mathrm{m}$ ),

$A(\lambda)=\frac{A_{V}}{R_{V}^{\prime}} \times\left[2.659\left(-1.857+\frac{1.040}{\lambda}\right)+R_{V}^{\prime}\right]$,

with $R_{V}^{\prime}=4.05$ and visual extinction $A_{V}$. 
G. Busch et al.: Star formation in NGC 1808

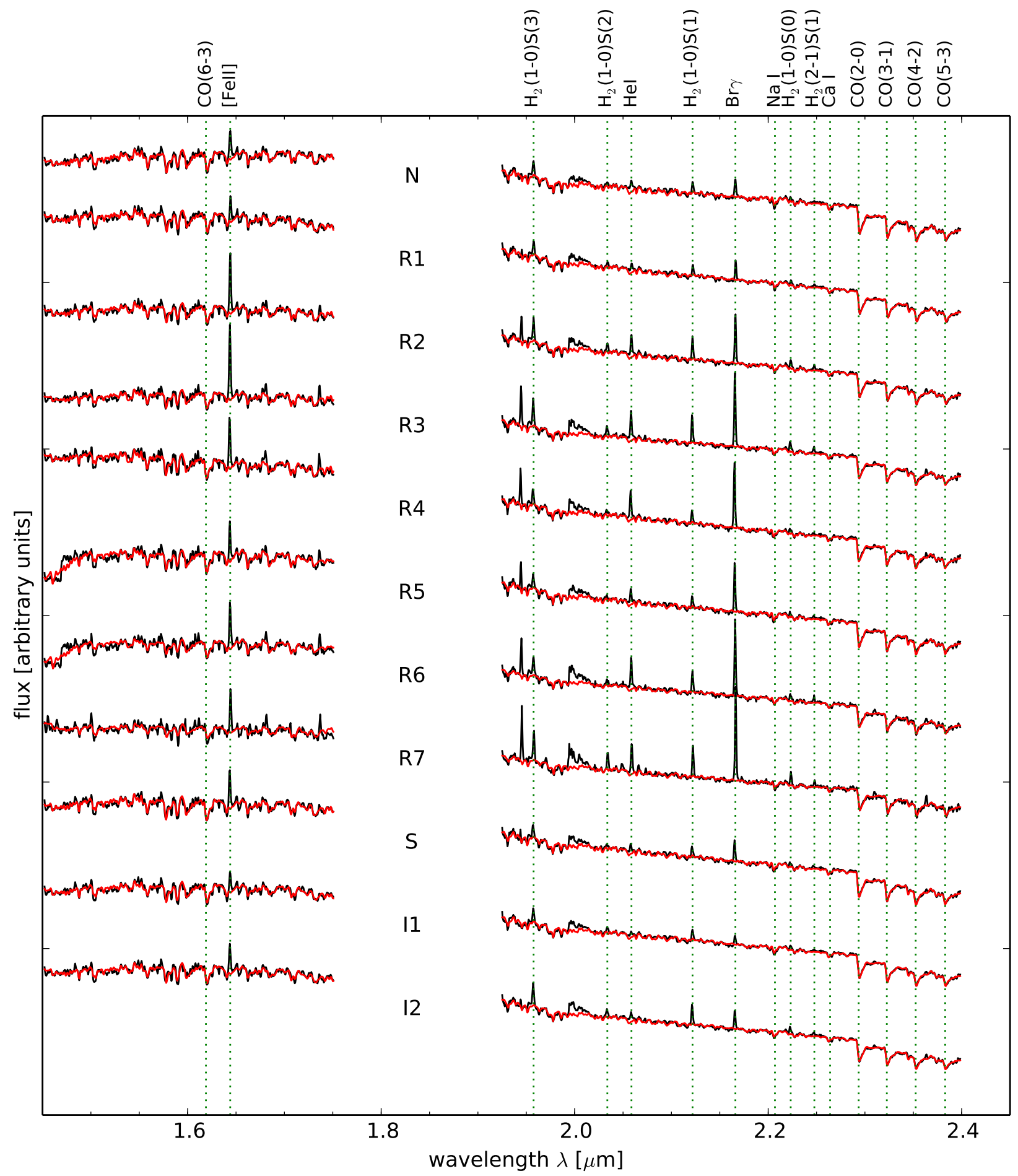

Fig. 5. Spectra of the nuclear regions plus seven apertures in the circumnuclear ring (R1-7), a possibly shocked region (S), and two apertures in the inter-ring region (I1-2). The positions where the apertures are extracted are indicated in Figs. 3 and 4. Several emission and absorption lines are indicated with green vertical lines. The stellar continuum fit is shown in red.

The internal gas extinction can now be estimated by comparing the observed flux ratio of two hydrogen recombination lines (in our case we use $\mathrm{Pa} \beta$ and $\mathrm{Br} \gamma$ ) with the theoretical ratio between these two lines. For a case $\mathrm{B}$ recombination scenario with a typical electron density of $n_{\mathrm{e}}=10^{4} \mathrm{~cm}^{-3}$ and a temperature of $10^{4} \mathrm{~K}$ this ratio is $\mathrm{Pa} \beta / \mathrm{Br} \gamma=5.89$ (Osterbrock \& Ferland 2006). The visual extinction is then given by

$A_{V}=11.52 \times \log \left(\frac{5.89}{F_{\text {obs, Pa } \beta} / F_{\text {obs, Br } \gamma}}\right)$.
Since the $J$-band data cube does not include the region around aperture R7, we cannot determine the extinction in this aperture. For all other apertures, we list the visual extinction $A_{V}$ in Table 1. The derived values of $A_{V}$ range from around 1.5 to 3.3 and are therefore a bit lower than but still consistent with the typical values from previous studies (Krabbe et al. 1994; Kotilainen et al. 1996; Rosenberg et al. 2012), which range from 2.5 to 5 .

In the left-hand panel of Fig. 6, we present the extinction map $\left(A_{V}\right.$ in mag) derived from the $\mathrm{Pa} \beta / \mathrm{Br} \gamma$ ratio as explained 
Table 1. Emission lines fluxes in the selected apertures, and derived quantities: extinction and ionised gas mass.

\begin{tabular}{cccccccc}
\hline \hline Aperture & $\begin{array}{c}\mathrm{Pa} \beta \\
\lambda 1.282 \mu \mathrm{m}\end{array}$ & $\begin{array}{c}{[\mathrm{Fe} \mathrm{II}]} \\
\lambda 1.644 \mu \mathrm{m}\end{array}$ & $\begin{array}{c}\mathrm{Pa} \alpha \\
\lambda 1.876 \mu \mathrm{m}\end{array}$ & $\begin{array}{c}\mathrm{Br} \delta \\
\lambda 1.945 \mu \mathrm{m}\end{array}$ & $\begin{array}{c}\mathrm{Br} \gamma \\
\lambda 2.166 \mu \mathrm{m}\end{array}$ & $\begin{array}{c}A_{V} \\
{[\mathrm{mag}]}\end{array}$ & $\begin{array}{c}\text { H II mass } \\
{\left[10^{5} M_{\odot}\right]}\end{array}$ \\
\hline $\mathrm{N}$ & $87.54 \pm 6.85$ & $35.41 \pm 4.00$ & $197.65 \pm 21.02$ & $5.89 \pm 0.95$ & $15.19 \pm 0.82$ & $3.3 \pm 0.3$ & 6.8 \\
$\mathrm{R} 1$ & $18.26 \pm 1.33$ & $5.13 \pm 0.53$ & $50.24 \pm 1.92$ & $1.16 \pm 0.13$ & $2.98 \pm 0.13$ & $2.0 \pm 0.2$ & 1.7 \\
$\mathrm{R} 2$ & $28.97 \pm 1.03$ & $8.70 \pm 0.37$ & $69.36 \pm 1.53$ & $2.96 \pm 0.10$ & $5.00 \pm 0.11$ & $2.7 \pm 0.1$ & 2.4 \\
$\mathrm{R} 3$ & $40.95 \pm 1.00$ & $10.02 \pm 0.32$ & $66.81 \pm 1.39$ & $4.48 \pm 0.11$ & $7.05 \pm 0.11$ & $2.8 \pm 0.1$ & 2.3 \\
$\mathrm{R} 4$ & $40.09 \pm 0.92$ & $6.59 \pm 0.39$ & $53.28 \pm 1.13$ & $3.84 \pm 0.13$ & $6.86 \pm 0.11$ & $1.5 \pm 0.1$ & 1.8 \\
$\mathrm{R} 5$ & $17.45 \pm 0.63$ & $5.86 \pm 0.44$ & $67.31 \pm 1.51$ & $3.34 \pm 0.12$ & $5.82 \pm 0.13$ & $1.7 \pm 0.1$ & 2.3 \\
$\mathrm{R} 6$ & $20.31 \pm 0.59$ & $4.35 \pm 0.25$ & $46.73 \pm 1.00$ & $2.70 \pm 0.05$ & $4.70 \pm 0.07$ & $2.9 \pm 0.1$ & 1.6 \\
$\mathrm{R} 7^{a}$ & - & $1.80 \pm 0.10$ & $52.50 \pm 0.60$ & $1.94 \pm 0.06$ & $3.59 \pm 0.05$ & - & 1.8 \\
$\mathrm{~S}$ & $19.43 \pm 1.37$ & $7.63 \pm 0.66$ & $29.96 \pm 1.36$ & $1.29 \pm 0.13$ & $3.32 \pm 0.15$ & $1.7 \pm 0.2$ & 1.0 \\
$\mathrm{I} 1$ & $11.73 \pm 1.55$ & $6.18 \pm 0.76$ & $22.67 \pm 1.63$ & $0.53 \pm 0.22$ & $1.94 \pm 0.18$ & $2.5 \pm 0.4$ & 0.8 \\
$\mathrm{I} 2$ & $15.00 \pm 1.02$ & $5.11 \pm 0.44$ & $25.53 \pm 1.17$ & $1.08 \pm 0.06$ & $2.39 \pm 0.10$ & $2.2 \pm 0.2$ & 0.9 \\
\hline
\end{tabular}

Notes. The emission line fluxes are corrected for extinction and given in units of $10^{-18} \mathrm{~W} \mathrm{~m}^{-2}$. All apertures have a radius of 0 .'75, which corresponds to a physical area of $6800 \mathrm{pc}^{2}$. ${ }^{(a)}$ Values are not corrected for extinction because extinction could not be determined in this aperture.

Table 2. Molecular hydrogen emission line fluxes in the selected apertures, and derived quantities: hot and cold gas mass, and cold gas density.

\begin{tabular}{ccccccccc}
\hline \hline Aperture & $\begin{array}{c}\mathrm{H}_{2}(1-0) \mathrm{S}(3) \\
\lambda 1.958 \mu \mathrm{m}\end{array}$ & $\begin{array}{c}\mathrm{H}_{2}(1-0) \mathrm{S}(2) \\
\lambda 2.034 \mu \mathrm{m}\end{array}$ & $\begin{array}{c}\mathrm{H}_{2}(1-0) \mathrm{S}(1) \\
\lambda 2.122 \mu \mathrm{m}\end{array}$ & $\begin{array}{c}\mathrm{H}_{2}(1-0) \mathrm{S}(0) \\
\lambda 2.223 \mu \mathrm{m}\end{array}$ & $\begin{array}{c}\mathrm{H}_{2}(2-1) \mathrm{S}(1) \\
\lambda 2.248 \mu \mathrm{m}\end{array}$ & $\begin{array}{c}\text { Hot } \mathrm{H}_{2} \text { mass } \\
{\left[M_{\odot}\right]}\end{array}$ & $\begin{array}{c}\text { Cold gas mass } \\
{\left[10^{6} M_{\odot}\right]}\end{array}$ & $\begin{array}{c}\log \left(\Sigma_{\text {gas }}\right) \\
{\left[M_{\odot} \mathrm{pc}^{-1}\right]}\end{array}$ \\
\hline $\mathrm{N}$ & $15.27 \pm 2.06$ & $3.50 \pm 1.49$ & $9.42 \pm 1.19$ & $2.51 \pm 1.33$ & $1.42 \pm 0.84$ & 78 & $24-125$ & 4.06 \\
$\mathrm{R} 1$ & $3.01 \pm 0.22$ & $0.85 \pm 0.22$ & $1.86 \pm 0.15$ & $0.78 \pm 0.17$ & $0.62 \pm 0.22$ & 15 & $5-25$ & 3.36 \\
$\mathrm{R} 2$ & $3.00 \pm 0.17$ & $1.09 \pm 0.13$ & $2.39 \pm 0.09$ & $0.91 \pm 0.12$ & $0.50 \pm 0.12$ & 20 & $6-32$ & 3.47 \\
$\mathrm{R} 3$ & $3.14 \pm 0.12$ & $1.27 \pm 0.14$ & $2.81 \pm 0.10$ & $1.05 \pm 0.08$ & $0.61 \pm 0.12$ & 23 & $7-37$ & 3.54 \\
$\mathrm{R} 4$ & $2.07 \pm 0.15$ & $0.80 \pm 0.20$ & $1.45 \pm 0.11$ & $0.65 \pm 0.12$ & $0.26 \pm 0.09$ & 12 & $4-19$ & 3.25 \\
$\mathrm{R} 5$ & $2.58 \pm 0.26$ & $0.52 \pm 0.23$ & $1.56 \pm 0.17$ & $0.47 \pm 0.17$ & $0.45 \pm 0.19$ & 13 & $4-21$ & 3.28 \\
$\mathrm{R} 6$ & $1.64 \pm 0.10$ & $0.65 \pm 0.15$ & $1.39 \pm 0.06$ & $0.50 \pm 0.07$ & $0.35 \pm 0.07$ & 12 & $3-18$ & 3.23 \\
$\mathrm{R} 7^{a}$ & $1.23 \pm 0.07$ & $0.77 \pm 0.07$ & $1.25 \pm 0.04$ & $0.57 \pm 0.03$ & $0.23 \pm 0.03$ & 10 & $3-17$ & 3.19 \\
$\mathrm{~S}$ & $3.06 \pm 0.27$ & $0.92 \pm 0.25$ & $1.96 \pm 0.19$ & $0.76 \pm 0.19$ & $0.62 \pm 0.16$ & 16 & $5-26$ & 3.38 \\
$\mathrm{I} 1$ & $4.04 \pm 0.31$ & $1.46 \pm 0.23$ & $2.46 \pm 0.23$ & $1.04 \pm 0.21$ & $0.65 \pm 0.24$ & 20 & $6-33$ & 3.48 \\
$\mathrm{I} 2$ & $3.77 \pm 0.20$ & $1.36 \pm 0.20$ & $2.81 \pm 0.14$ & $0.98 \pm 0.09$ & $0.65 \pm 0.18$ & 23 & $7-37$ & 3.54 \\
\hline
\end{tabular}

Notes. The emission line fluxes are corrected for extinction and given in units of $10^{-18} \mathrm{~W} \mathrm{~m}^{-2}$. All apertures have a radius of 0 '?75, which corresponds to a physical area of $6800 \mathrm{pc}^{2}$. ${ }^{(a)}$ Values are not corrected for extinction because extinction could not be determined in this aperture.

above. We see that the extinction in the nucleus and in some of the spots along the ring is quite high, ranging from a few up to 5 mag, indicating that they might be dust-enshrouded starbursts. Furthermore, there are two regions ("I1" south of the nucleus and the region north of the nucleus) that show enhanced extinction of about 3 mag. The extinction map is consistent with the $H-K$ colour map and the optical HST image (Fig. 6, middle and right panel), that is dusty regions (e.g. between R2 and R3, and from R5 to R6 and R7) show higher extinction.

\subsubsection{Diagnostic line ratios}

In the left panel of Fig. 7, we show a map of the line ratio $\log ([\mathrm{Fe} \mathrm{II}] / \mathrm{Br} \gamma)$ that can be used to analyse the $[\mathrm{Fe} \mathrm{II}]$ excitation mechanism. In this plot and the next, we also show the $3.6 \mathrm{~cm}$ radio-continuum image of Collison et al. (1994) (taken from Kotilainen et al. 1996) as contours. Low values in the line ratio $\log ([\mathrm{Fe} \mathrm{II}] / \mathrm{Br} \gamma)$ in the circumnuclear ring (spots $\mathrm{R} 1-\mathrm{R} 7)$ are typical for starbursts. On the other hand, in the regions $\mathrm{S}$ and I1-2 and in the region to the north-west of the FOV, shocks could be present since they show rather high values. The suspected star formation regions with low line ratios and the suspectedly shocked regions with high line ratios both show high radiocontinuum fluxes.

In the right-hand panel, we show a map of the line ratio $\log \left(\mathrm{H}_{2} \lambda 2.12 \mu \mathrm{m} / \mathrm{Br} \gamma\right)$. In the ring, we find very low ratios down to -0.6 , that are indicative of starbursts. In the nuclear region, however, we find values that are consistent with AGN excitation. On the western side, between the nucleus and the ring, high line ratios that are indicative of shock excitation are found.

\subsection{Stellar kinematics}

In order to trace the stellar kinematics, i.e. the stellar line-ofsight velocity (LOSV) and the stellar velocity dispersion $\left(\sigma_{*}\right)$, we fit the region containing the $\mathrm{CO}$ band heads at around $2.3 \mu \mathrm{m}$. Since stellar absorption features generally show a lower signalto-noise ratio $(\mathrm{S} / \mathrm{N})$ than strong emission lines whose emission line flux maps we plot below, we need to bin the data. Only then do we reach sufficient $\mathrm{S} / \mathrm{N}$ in all parts of the FOV for a reliable fit. The Voronoi binning method is an adaptive smoothing algorithm which bins the data to a constant $\mathrm{S} / \mathrm{N}$ while preserving 

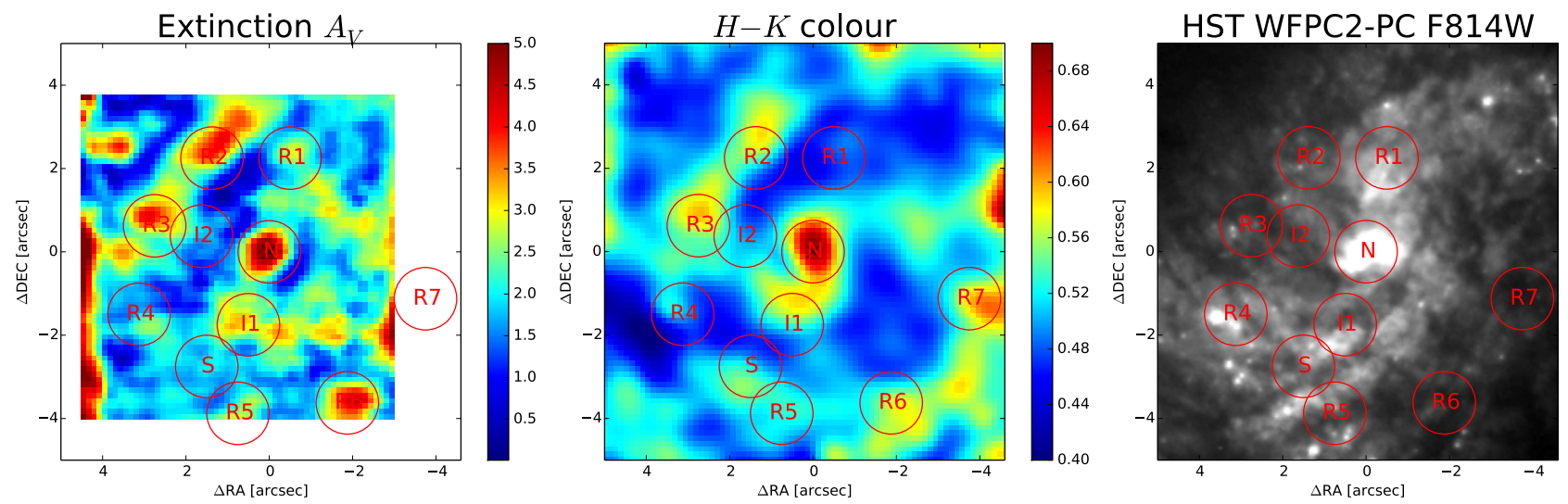

Fig. 6. From left to right: map of the visual extinction $\left(A_{V}\right)$ in mag (derived from the line ratio $\mathrm{Pa} \beta / \mathrm{Br} \gamma$ ), the $H-K$ colour, and a HST $F 814 W$ image. The nucleus is denoted by a cross. The apertures introduced in Sect. 3.1 are marked with red circles for orientation.
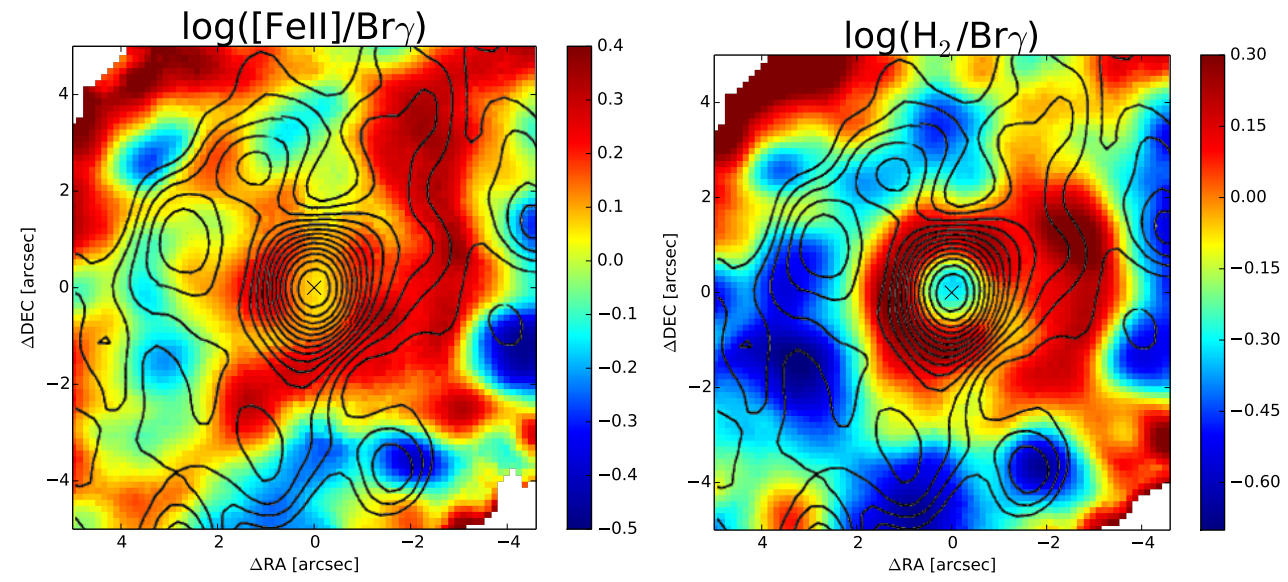

Fig. 7. From left to right: the emission line ratios $\log ([\mathrm{Fe} \mathrm{II}] / \mathrm{Br} \gamma)$ and $\log \left(\mathrm{H}_{2} \lambda 2.12 \mu \mathrm{m} / \mathrm{Br} \gamma\right)$. The nucleus is denoted by a cross. We overlay the contours of the $3.6 \mathrm{~cm}$ radio-continuum image.

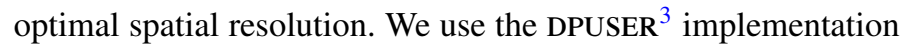
of the code originally provided by Cappellari \& Copin (2003).

For the fit, we use the Penalized Pixel-Fitting method (pPXF, Cappellari \& Emsellem 2004). As stellar template spectra, we take the Gemini Spectral Library of Near-IR Late-Type Stellar Templates (Winge et al. 2009) which consists of 29 giant and supergiant stars with spectral classes from F7III to M3III that have been observed with the IFU of the GEMINI integral-field spectrograph GNIRS.

A map of the stellar velocity dispersion is presented in Fig. 8. It shows values from around $60 \mathrm{~km} \mathrm{~s}^{-1}$ to $110 \mathrm{~km} \mathrm{~s}^{-1}$. The centre shows a "dip" with a lower velocity dispersion of only around $60 \mathrm{~km} \mathrm{~s}^{-1}$. Furthermore, the map shows a ring-like structure with lower velocity dispersion of around $70 \mathrm{~km} \mathrm{~s}^{-1}$. This ring structure has a radius of about $4^{\prime \prime}(\approx 240 \mathrm{pc})$. Assuming intrinsic circular shape, we get an inclination of $i=50^{\circ}-60^{\circ}$ from the eccentricity.

The stellar velocity field is shown in the left panel of Fig. 9. It shows a clear rotation pattern with redshift to the north-west and blueshift to the south-east. Maximum velocities are of the order of $100-120 \mathrm{~km} \mathrm{~s}^{-1}$. Assuming that the spiral arms seen in the optical images in Figs. 1 and 3 are trailing, the near side of the disc is in the south-west. The isovelocity lines show an S-shape

\footnotetext{
3 DPUSER was written by Thomas Ott (MPE Garching) as a software package for reducing astronomical speckle data. http://www.mpe. mpg.de/ ott/dpuser/index.html
}

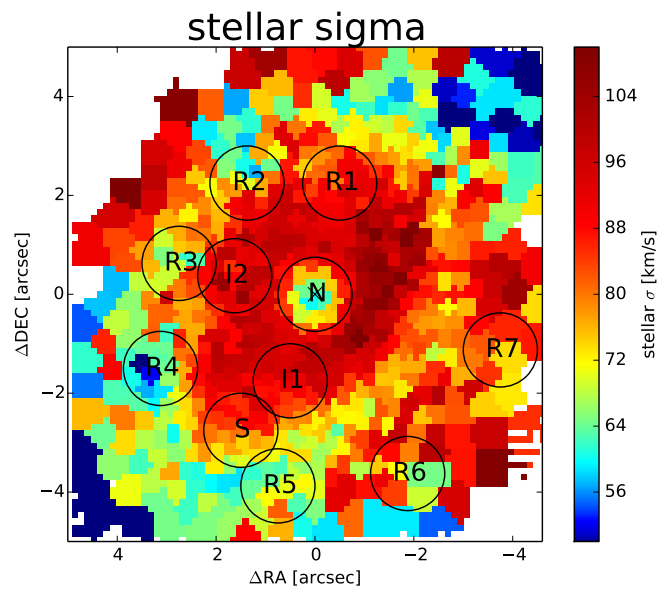

Fig. 8. Stellar velocity dispersion obtained by fitting the region around the $\mathrm{CO}$ band heads at $2.3 \mu \mathrm{m}$.

that is indicative of a disturbance of the velocity field, e.g. by a nuclear bar. This feature is discussed below.

\subsection{Gas kinematics}

The gas kinematics gives insight into the overall dynamics of the host galaxy in comparison to the stellar kinematics. In addition 

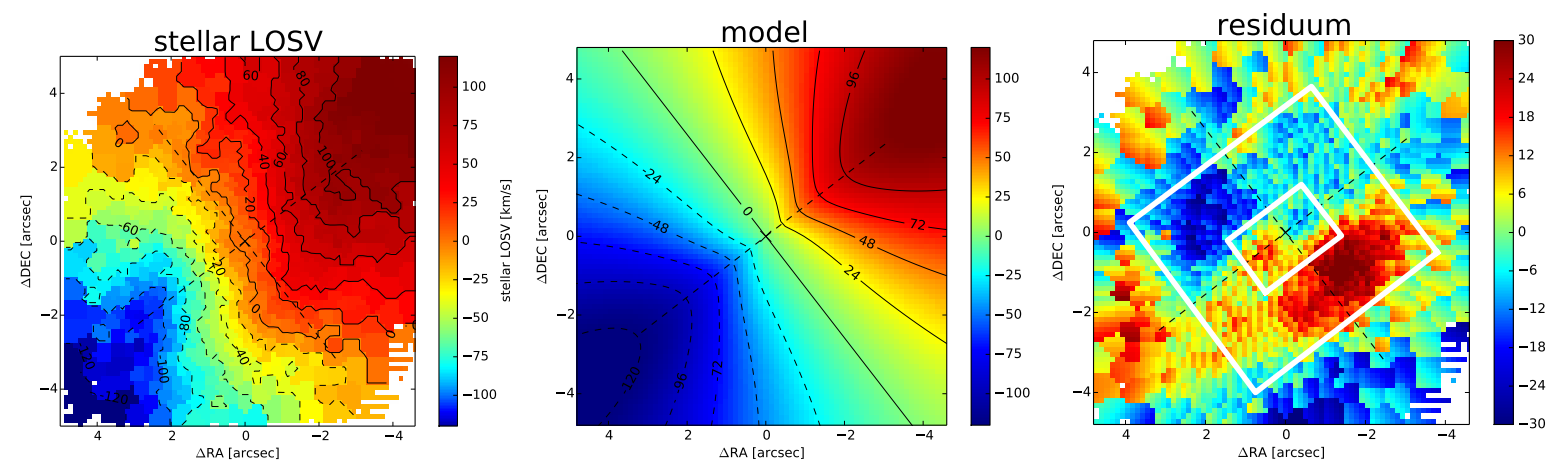

Fig. 9. Left: stellar line-of-sight velocity obtained by fitting the region around the CO band heads at $2.3 \mu \mathrm{m}$. Middle: rotating disc model fitted to the stellar velocity field. Right: residual between LOSV and model. The residual shows sub-structures that are indicated with white boxes. The cross denotes the peak of the $K$-band continuum emission, the black dashed lines show the line of nodes and the zero-velocity line of the model.

the gas kinematics allows us to find and study zones of enhanced activity (due to the nucleus or due to star formation) via regions of enhanced velocity dispersion or excitation.

The fit routine that we use in Sect. 3.1 to derive the emission line flux distributions also delivers central positions and widths of the emission line fits from which we can derive the LOSV and velocity dispersion $(\sigma)$.

In Fig. 10, we show the line-of-sight velocity fields of $\operatorname{Br} \gamma$, $\mathrm{H}_{2} \lambda 2.12 \mu \mathrm{m}$, and [Fe II] $\lambda 1.644 \mu \mathrm{m}$. The systemic velocity of $1056.8 \mathrm{~km} \mathrm{~s}^{-1}$ (derived in Sect. 4.1) has been subtracted. The maps for $\mathrm{Br} \gamma$ and $\mathrm{H}_{2} \lambda 2.12 \mu \mathrm{m}$ have been derived from the $K$-band cube, which has higher spectral resolution, whereas the [Fe II] map has been derived from the $H+K$-cube (but we use the $H+K$-data to cross-check the maps derived from $K$-band cubes). All velocity maps show redshifts to the north-west and blueshift to the south-east, in accordance with the stellar kinematics. However, the velocity fields of the gas show more pronounced S-shaped zero-velocity contours than the stellar field. Furthermore, the strong velocity gradient to the north-west from the nucleus is striking. While the $\operatorname{Br} \gamma$ and the [Fe II] velocity fields are similar, the $\mathrm{H}_{2}$ field differs from them. The centre shows a redshift of around $40 \mathrm{~km} \mathrm{~s}^{-1}$ (after subtraction of the systemic velocity) and shows redshifted gas in elongated features reaching from the west to the nucleus, as well as an elongated feature along the north-west to south-east axis around $3^{\prime \prime}$ south of the nucleus. These features are discussed in a later section.

In Fig. 11, we show the velocity dispersion fields for the same emission lines. The maps have been corrected for the instrumental broadening $\left(33 \mathrm{~km} \mathrm{~s}^{-1}\right.$ for the $K$-band maps $\mathrm{Br} \gamma$ and $\mathrm{H}_{2} \lambda 2.12 \mu \mathrm{m}, 97 \mathrm{~km} \mathrm{~s}^{-1}$ for the $H+K$-band map [Fe II] $\lambda 1.644 \mu \mathrm{m})$. In $\mathrm{Br} \gamma$, the velocity dispersion shows maximum values of $\sim 100 \mathrm{~km} \mathrm{~s}^{-1}$ at around $0 \prime 9$ to the east and west of the nucleus. In the centre, the velocity dispersion drops down to $\sim 80 \mathrm{~km} \mathrm{~s}^{-1}$. The molecular hydrogen line $\mathrm{H}_{2} \lambda 2.12 \mu \mathrm{m}$ shows the maximum velocity dispersion values of $\sim 80 \mathrm{~km} \mathrm{~s}^{-1}$ in a ringlike shape, with a connection to the centre. This structure lies inside the circumnuclear star-forming ring which is visible in $\mathrm{Br} \gamma$ and $\operatorname{Pa} \alpha$ emission. The highest values are seen in a region to the south-east of the centre, between spots I1 and S with values of $\sim 100 \mathrm{~km} \mathrm{~s}^{-1}$. The velocity dispersion of [Fe II] $\lambda 1.644 \mu \mathrm{m}$ peaks close to the centre $(\sim 0$ ! 7 to the east $)$ with values of $\sim 110 \mathrm{~km} \mathrm{~s}^{-1}$. Another peak is seen in the region where the velocity dispersion of the $\mathrm{H}_{2} \lambda 2.12 \mu \mathrm{m}$ line also peaks, between the spots $\mathrm{I} 1$ and $\mathrm{S}$ $\left(\sim 70 \mathrm{~km} \mathrm{~s}^{-1}\right)$. Additional regions with enhanced velocity dispersion are found between the spots R6 and R7, and outside the mentioned $\mathrm{Br} \gamma$ ring to the east side, particularly in the north-east. However, these regions are at the edges of the field-of-view and the signal-to-noise ratio is therefore much lower. Interestingly, we find that the velocity dispersion in the star formation ring and the radio hot spots is relatively low, indicating that the gas in these regions is rather undisturbed.

\section{Discussion}

\subsection{Stellar kinematics}

The stellar velocity field suggests rotation (see the left panel of Fig. 9). Assuming circular orbits, we fit a Plummer gravitational potential, in which the velocity distribution is given by

$v_{r}=v_{\mathrm{s}}+\sqrt{\frac{R^{2} G M}{\left(R^{2}+A^{2}\right)^{3 / 2}}} \frac{\sin (i) \cos \left(\Psi-\Psi_{0}\right)}{\left[\cos ^{2}\left(\Psi-\Psi_{0}\right)+\frac{\sin ^{2}\left(\Psi-\Psi_{0}\right)}{\cos ^{2}(i)}\right]^{3 / 4}}$,

where $v_{\mathrm{s}}$ is the systemic velocity, $R$ and $\Psi$ are the cylindric coordinates of each pixel in the projected plane of the sky, $G$ is the gravitational constant, $M$ is the mass inside $R, A$ is the projected scale length of the disc, $i$ is the inclination of the disc, and $\Psi_{0}$ is the position angle of the line of nodes (e.g. Barbosa et al. 2006; Smajić et al. 2015). The inclination based on the D25 diameter in the blue band is $i=53^{\circ}$ (de Vaucouleurs et al. 1991, from the NED database). Based on the HST optical data in the centre, we estimate an inclination of $i \approx 52^{\circ}$. In the fit, we fix the inclination to the latter value to lower the number of free parameters.

The position angle of the line of nodes $\Psi=(128 \pm 1)^{\circ}$ is lower than the photometric position angle $133^{\circ}$ (de Vaucouleurs et al. 1991, from the NED database). All parameters have typical values for nearby galaxies, for example compared to the galaxies analysed in Barbosa et al. (2006). The systemic velocity $v_{\mathrm{s}}=$ $(1056.8 \pm 0.4) \mathrm{km} \mathrm{s}^{-1}$ is slightly higher than $995 \mathrm{~km} \mathrm{~s}^{-1}$ derived from H I measurements (Koribalski et al. 2004). We subtract the systemic velocity from the line-of-sight velocity maps of gas and stars in Figs. 8 and 10.

The stellar velocity field in Fig. 9 shows a clear deviation from pure rotation. When subtracting the rotating disc model from the stellar velocity field, we find systematic residuals (indicated by white boxes). On scales of $\sim 3^{\prime \prime}$, we see peaks in redshift in the south-west and in blueshift in the north-east. These structures occur because the S-shaped zero-velocity line is not included in the simple rotational model. S-shaped zerovelocity lines are a well-known signature for non-circular velocity fields (e.g. Combes et al. 2004; Emsellem et al. 2006; Riffel \& Storchi-Bergmann 2011), that are disturbed, for example by a bar potential (the position of the nuclear bar is indicated in the $K$-band continuum image in Fig. 2). 

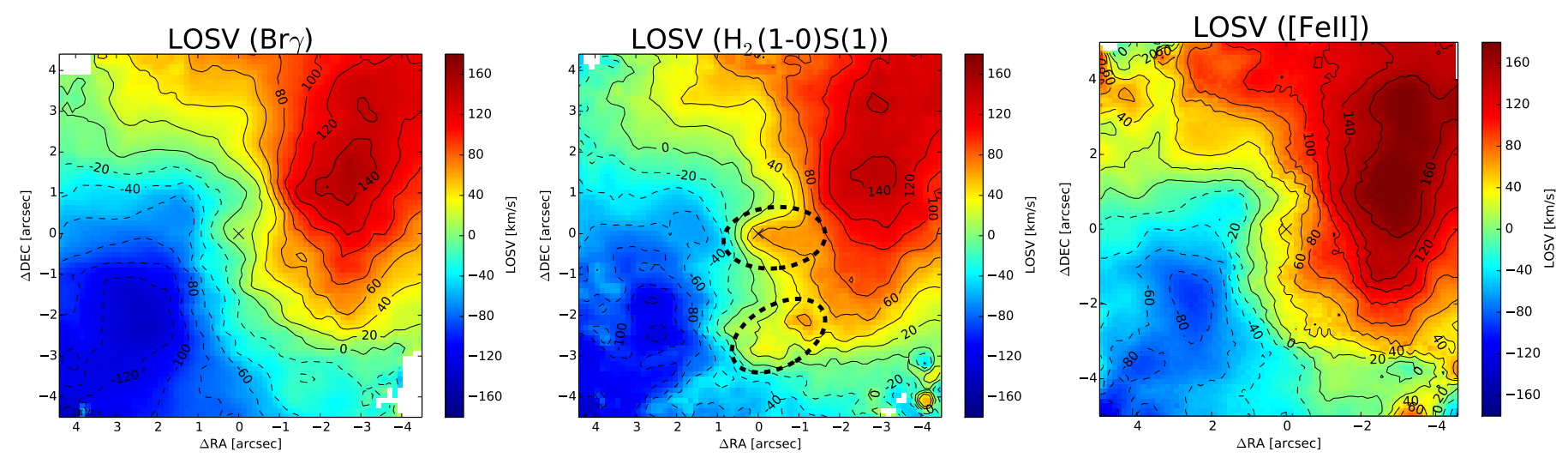

Fig. 10. Line-of-sight velocity maps of ionised gas $(\mathrm{Br} \gamma)$, molecular gas $\left(\mathrm{H}_{2} \lambda 2.12 \mu \mathrm{m}\right)$, and partially ionised gas ([Fe II] $\left.\lambda 1.644 \mu \mathrm{m}\right)$. The cross denotes the centre, i.e. the peak of the continuum emission. In the $\mathrm{H}_{2}$ LOSV-map, two elongated features are marked with dashed black ellipses.
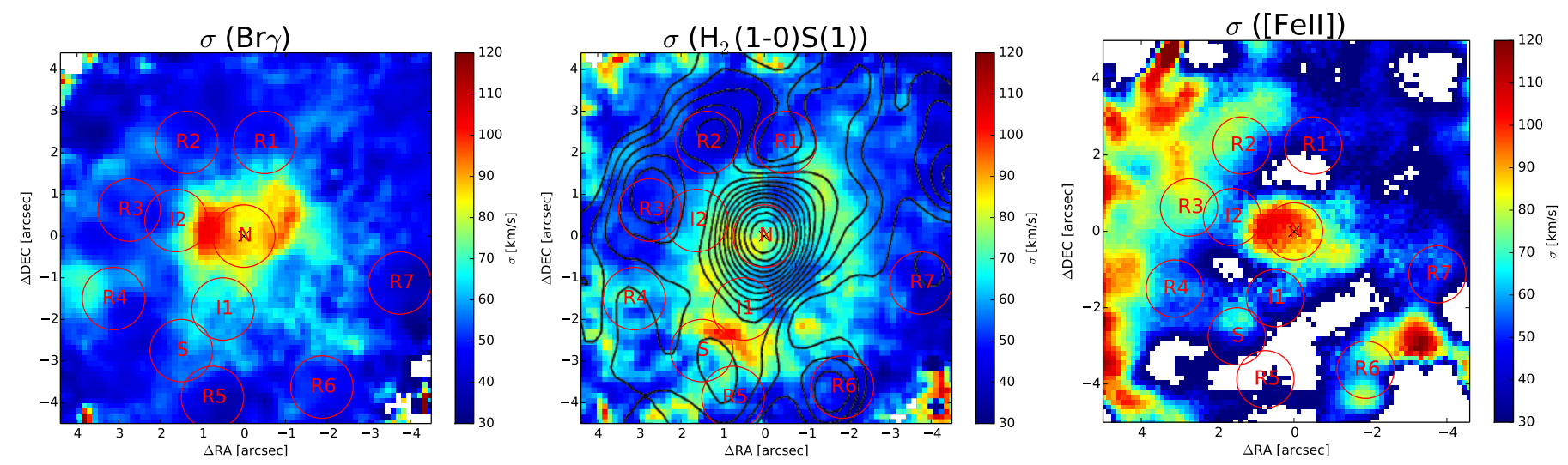

Fig. 11. Velocity dispersion maps of ionised gas (Br $\gamma)$, molecular gas $\left(\mathrm{H}_{2} \lambda 2.12 \mu \mathrm{m}\right)$, and partially ionised gas ([Fe II] $\left.\lambda 1.644 \mu \mathrm{m}\right)$. The cross denotes the centre, i.e. the peak of the continuum emission. In the map of molecular hydrogen, we overlay the radio contours.

Central drops in the velocity dispersion can indicate decoupled dynamics in the nucleus, e.g. by a nuclear disc. Falcón-Barroso et al. (2006) find that a significant fraction of spiral galaxies in the SAURON survey show that this feature and kinematically decoupled components are actually a common phenomenon. A central $\sigma$-drop in NGC 1808 was found by Emsellem et al. (2001) in ISAAC-longslit spectra, and is visible for the first time in its full 2D extent in our maps (Fig. 8). The most likely scenario, which is supported by numerical simulations and model calculations, is that these velocity drops are produced by young stars that form from cold gas with low velocity dispersion compared to the underlying old stellar population. Since they are more massive and brighter than the old stars, they will dominate the observed kinematics at near-infrared wavelengths (Emsellem et al. 2001; Wozniak et al. 2003; Comerón et al. 2008). This scenario is indeed consistent with our observations of young stellar clusters in the centre (see in particular Sect. 4.3.1 and 4.5.1). In addition to the central $\sigma$-drop, the circumnuclear ring (particularly the apertures R2-R5) show a lower velocity dispersion than the surroundings. This is indicative of younger stellar population in these regions (e.g. Riffel et al. 2011; Mazzalay et al. 2014).

Tight relations between the stellar velocity dispersion of the bulge and the mass of the central black hole have been found and interpreted in the context of galaxy evolution (Ferrarese \& Merritt 2000; Gebhardt et al. 2000). Here, we use the stellar velocity dispersion $\sigma_{*}$ to get a rough estimate of the mass of the central black hole. Running PPXF on the spectrum integrated over an aperture of $r=4^{\prime \prime}$ (using the settings described in Sect. 3.3), we obtain $\sigma_{*}=(105 \pm 5) \mathrm{km} \mathrm{s}^{-1}$. This value is lower than the value in the HyperLeda database ${ }^{4} \sigma_{*}=$ $(141.3 \pm 8.7) \mathrm{km} \mathrm{s}^{-1}$ and the values derived from fitting the CaT by Garcia-Rissmann et al. (2005), $\sigma_{*}=(119 \pm 6) \mathrm{km} \mathrm{s}^{-1}$ using the direct fitting method and $\sigma_{*}=(129 \pm 4) \mathrm{km} \mathrm{s}^{-1}$ using the cross-correlation method. However, Riffel et al. (2015a) find a systematically lower $\sigma_{*}$ for spiral galaxies when measured at the $K$-band $\mathrm{CO}$ band heads $\left(\sigma_{\mathrm{CO}}\right)$ than when using optical measurements $\left(\sigma_{\text {opt }}\right)$. Using their best fit, we find that our measurement corresponds to $\sigma_{\mathrm{opt}} \approx 135 \mathrm{~km} \mathrm{~s}^{-1}$, which is consistent with the optical measurements. Since most $M_{\mathrm{BH}}-\sigma_{*}$ relations in the literature are based on optical measurements, we use the latter value for the black hole mass estimates. Applying different relations, we get the following estimates: $\log \left(M_{\mathrm{BH}} / M_{\odot}\right)=7.7$ (Kormendy \& Ho 2013), $\log \left(M_{\mathrm{BH}} / M_{\odot}\right)=7.4$ (Gültekin et al. 2009), and $\log \left(M_{\mathrm{BH}} / M_{\odot}\right)=7.3$ and $\log \left(M_{\mathrm{BH}} / M_{\odot}\right)=7.0($ Graham \& Scott 2013 , using the relation for non-barred or barred galaxies, respectively). The difference between the values seems large, but it is within the scatter of the BH mass-host scaling relations (which is between 0.29 and 0.44 dex for the cited relations). We conclude that the black hole mass is of the order of a few $10^{7} M_{\odot}$.

However, two caveats have to be kept in mind. First, owing to the complicated $\sigma$ structure, in particular the central $\sigma$-drop, it is not completely clear which value is representative of the bulge's stellar velocity dispersion. One could argue that the central pixels have to be excluded because of their decoupled kinematics. However, the extent of the structure is relatively small

4 http://leda.univ-lyon1.fr/ (Makarov et al. 2014). 

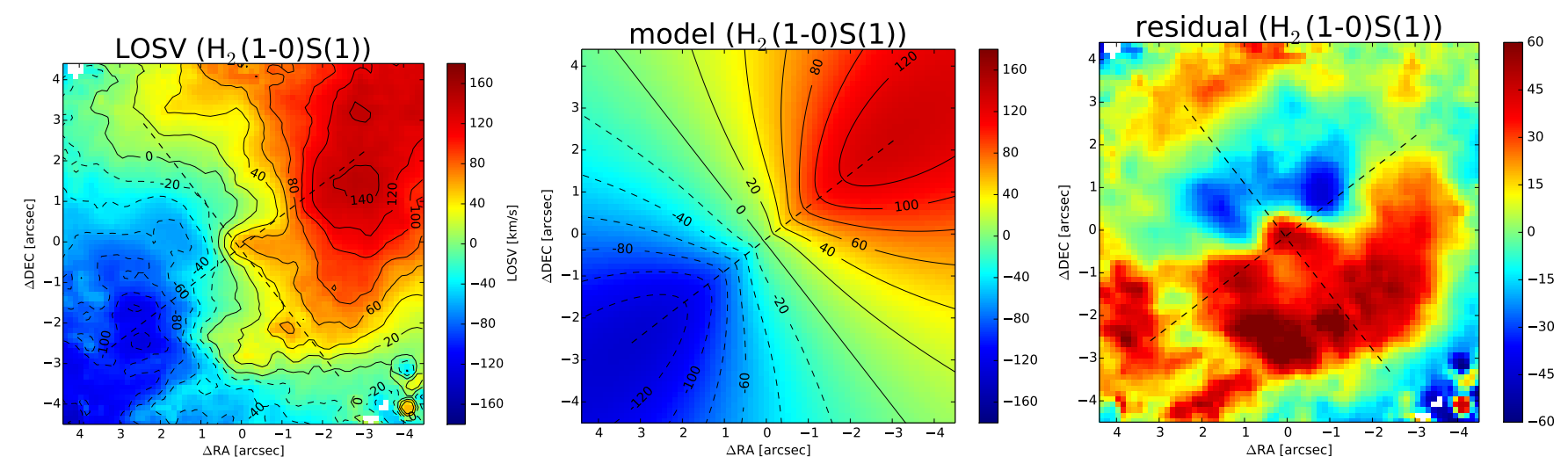

Fig. 12. From left to right: line-of-sight velocity map of $\mathrm{H}_{2}$ emission line, pure rotational model, and residual.

and does not significantly affect the luminosity weighted average. Second, central drops in the stellar velocity dispersion, as well as the dusty structure in the centre (see Figs. 3 and 6), are commonly seen as indicative of a pseudobulge structure that is the result of secular evolution (e.g. Fisher \& Drory 2016). It is under discussion whether pseudobulges follow $M_{\mathrm{BH}}$-host correlations (Kormendy et al. 2011), which would put into question our black hole mass estimates.

\subsection{Gaseous kinematics}

The velocity fields of the gas in the central $200 \mathrm{pc}$ show more obvious deviations from pure rotation than the stellar velocity field (see Fig. 10). In particular, a strong S-shaped zero-velocity line is striking. A likely explanation for this feature is the presence of non-circular motions, such as oval flows, secondary bars, or warps whose axes are not parallel to the symmetry axes of the large-scale velocity field (e.g. Maciejewski et al. 2002; Mazzalay et al. 2014; Davies et al. 2014a). To subtract the regular velocity field, we performed the same fits as in Sect. 4.1. We note that the gas velocity fields have a slightly higher redshift than the stars and, furthermore, the scale length is smaller, which means that the gas is more centrally concentrated, particularly the $[\mathrm{Fe}$ II] emission. Despite the clear twist in the centres, the position angles of the lines of nodes of gas and stellar velocity fields agree on larger scales (within a few degrees), which shows that the velocity fields are aligned. To better compare stellar and gaseous kinematics, we then fix most of the parameters (centre, inclination, position angle) to the results of the stellar fit. In Fig. 12, we show the resulting model and the residual based on the $\mathrm{H}_{2}$ kinematics. The deviations from circular motions visible in the residua show similar structures and - more importantly the same sign (though not the same amplitude), which indicates that they are produced by the same phenomenon (probably noncircular motions due to the bar which are more amplified in the gas than in the stars).

\subsubsection{Inflow or outflow?}

In the following we compare stellar and gaseous kinematics. It is known that in many galaxies the rotation velocity of gas has a higher amplitude than that of the stars. This occurs because a significant fraction of the kinetic energy of the stars can go into random motions (velocity dispersion), while gas is more confined to the galaxy plane. We therefore compare the rotation curve (Fig. 13) of gas and stars. We find that in the south-east, the rotation curves do not show the aforementioned behaviour, but

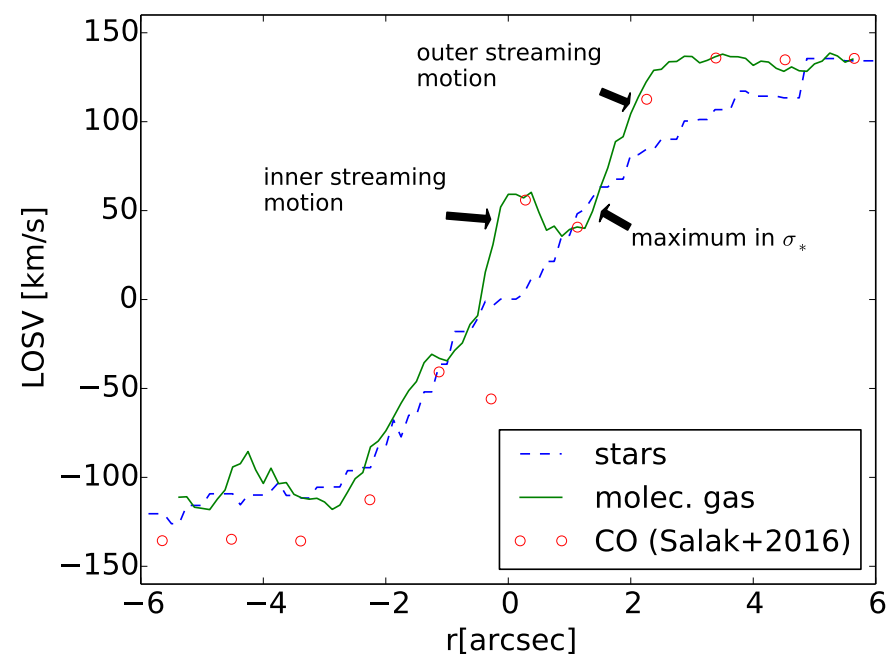

Fig. 13. Line-of-sight velocity cut from south-east to north-west along the line of nodes. The rotation curves of stars and hot molecular gas are derived from the SINFONI data. Data points for cold gas (CO) are added from Salak et al. (2016).

are well aligned. In the north-west, two bumps are seen that coincide with features in the residuals. At the radius of the ring with high stellar velocity dispersion (see Fig. 8), the velocities are very similar. This is contrary to the expectation that the difference between gas and stellar velocity should be largest where the stellar velocity dispersion is largest. We suspect that the velocity amplitudes are not very different in this case because the overall stellar velocity dispersion of the central component is quite low, probably owing to the presence of a pseudobulge. In this case the kinematics of the stars in the centre would be dominated by rotation and not velocity dispersion (which would be the case in "classical" bulges).

We also add the rotation curve of the cold gas that Salak et al. (2016) derive from their CO measurements ${ }^{5}$. The rotation curves show a comparable degree of rotation. In the south-east, where the rotation curves deviate most, there are also residuals of the order of $+50 \mathrm{~km} \mathrm{~s}^{-1}$ visible in their residual (their Fig. 29d).

We conclude that hot and cold gas, as well as stars, all show a very similar degree of rotation and therefore a simple residual map, which we obtain by subtracting the stellar velocity field

\footnotetext{
5 The data points are derived from their rotational model, not the observed data, and are listed in their Table 6. We multiply the inclination corrected values by $\sin (i)$ to compare them with our data.
} 
from the velocity fields of $\mathrm{Br} \gamma$ and $\mathrm{H}_{2}$ (Fig. 14, left and middle panel), can give us an estimate of the non-rotational motions in the gas velocity fields.

The first prominent feature is a residual redshift around $3^{\prime \prime}$ to the south-west which shows a partial ring structure (marked with the dashed lines in Fig. 14). We note that this feature does not coincide with the star formation ring, but resembles the region inside this ring. In the line-ratio maps (Fig. 7) this region shows high [ $\mathrm{Fe}$ II] $/ \mathrm{Br} \gamma$ and $\mathrm{H}_{2} / \mathrm{Br} \gamma$ line ratios which could indicate shocks by inflowing gas. In their ${ }^{12} \mathrm{CO}(J=1-0)$ map, which traces the cold molecular gas, Salak et al. (2016) find a spatially coincident streaming motion inside the gaseous nuclear spiral arm with a magnitude of $\sim 50 \mathrm{~km} \mathrm{~s}^{-1}$, which is fully consistent with our measurement, indicating that we trace the same motion in warm and cold molecular gas.

The second prominent feature is the nuclear two-arm spiral structure in the central $100 \mathrm{pc}$ (marked with solid lines), which is only detectable in our near-infrared data thanks to the higher spatial resolution of $<1^{\prime \prime}(\sim 50 \mathrm{pc})$. Assuming that they are located within the disc plane and that the near side of the disc is in the south-west (see Sect. 3.3), we conclude that the residual spiral arms could correspond to streaming motions towards the centre. Compared to the optical HST F614W map (Fig. 14, right panel), we see that the possible inflow motions coincide with dust features. Davies et al. (2014a) find that these chaotic circumnuclear dust structures are typically associated with external accretion in groups of galaxies.

To quantify the amount of cold gas that follows these streams and to calculate the resulting gravity torques (e.g. García-Burillo et al. 2005), upcoming high-angular resolution sub-mm observations with ALMA will be helpful.

The velocity dispersion of [Fe II] shows enhanced values in the central region (Fig. 11). This is likely caused by shocks due to supernova of an aging starburst. There is no indication for a jet that could be responsible for the shock. Furthermore, the velocity dispersion is enhanced in region $\mathrm{S}$ and all the way to the centre, i.e. where the residual line-of-sight velocity of $\mathrm{H}_{2}$ (Fig. 14) shows high redshifts. A possible explanation is that inflowing molecular gas shocks the interstellar medium, resulting in a higher flux level and velocity dispersion in the [Fe II] emission line.

In addition to the aforementioned features on $100 \mathrm{pc}$ scales, a possible outflow or superwind in NGC 1808 on kpc scale has been discussed for long time. Dust lanes detected in optical images indicate the presence of a gas outflow (Phillips 1993). This is supported by the detection of forbidden line ([O III], [N II], [S II]) emission and high [N II]/H $\alpha$ line ratios, indicative of shocked gas, at the base of the suspected outflow, $\gtrsim 1 \mathrm{kpc}$ north-east of the centre (Sharp \& Bland-Hawthorn 2010). Salak et al. (2016) measure high velocity dispersion in this region and can even separate the $\mathrm{CO}$ emission lines into two components with a separation of $\gtrsim 100 \mathrm{~km} \mathrm{~s}^{-1}$. They further subtract a rotating disc model from the gas velocity field, resulting in blueshifted emission. Assuming an outflow perpendicular to the disc, the blueshifted velocity translates into a deprojected outflow velocity of $\sim 180 \mathrm{~km} \mathrm{~s}^{-1}$. On the other hand, assuming motions within the plane of the disc, the blueshifted emission would correspond to an inflow with $\sim 120 \mathrm{~km} \mathrm{~s}^{-1}$ which is similar to the rotational velocity. This is rather unlikely and therefore the authors favour the outflow scenario.

Since our FOV covers only the central $10^{\prime \prime} \times 10^{\prime \prime}$, we have only limited information about the suspected outflow region. Consistent with the mentioned previous observations, we find increased velocity dispersion of $\mathrm{H}_{2}$ emission lines in the north-east corner (Fig. 11, $\sim 200 \mathrm{~km} \mathrm{~s}^{-1}$ compared to $\sim 100 \mathrm{~km} \mathrm{~s}^{-1}$ in the star-forming ring). However, we cannot separate two components with the available spectral resolution. Furthermore, the line ratios of $\mathrm{H}_{2} / \mathrm{Br} \gamma$ and $[\mathrm{Fe} \mathrm{II}] / \mathrm{Br} \gamma$ have high values in this region, which is indicative of shocked gas. Unfortunately, this region is at the edge of our FOV and has low signal-to-noise. Further near-infrared IFS observations focused on the suspected outflow region are desirable.

In addition, based on our near-infrared data, we do not detect clear indications of this outflow in the central region $\left(|r| \lesssim 5^{\prime \prime} \approx\right.$ $300 \mathrm{pc}$ ), which raises the questions of where exactly the outflow starts and what the driver is.

\subsection{Gaseous excitation}

\subsubsection{Near-infrared diagnostic diagram}

Diagnostic diagrams use line ratios of diagnostic lines to determine the dominating excitation mechanisms of the line-emitting gas. They are a very useful tool in the optical where they were established (e.g. Baldwin et al. 1981; Kewley et al. 2001; Kauffmann et al. 2003; Schawinski et al. 2007; Bremer et al. 2013; Davies et al. 2014b; Vitale et al. 2015).

The success of diagnostic diagrams in the optical motivated the search for similar tools in the near-infrared. Here, line ratios between star formation tracers (such as $\mathrm{Pa} \alpha, \mathrm{Pa} \beta, \mathrm{Br} \gamma$ ) and shock tracers (such as different emission lines of $\mathrm{H}_{2}$ or [Fe II]) are used to find the dominating source of excitation. One example is the line ratio $\mathrm{H}_{2} \lambda 2.122 \mu \mathrm{m} / \mathrm{Br} \gamma$. The lowest values are found in starburst regions where young OB stars produce strong emission in the hydrogen lines (e.g. Br $\gamma$ ). Higher values occur for older stellar populations where the number of $\operatorname{Br} \gamma$ emitting young OB stars has declined, but the rate of supernovae has increased. Supernovae produce shocks that can be traced by shock tracing near-infrared lines such as $\mathrm{H}_{2}$ or [Fe II]. The line ratio $\mathrm{H}_{2} \lambda 2.122 \mu \mathrm{m} / \mathrm{Br} \gamma$ shows typical values of $\lesssim 0.4$ for star formation, low-ionisation nuclear emission line regions (LINERs) show values of $\gtrsim 0.9$, while Seyferts typically have values in between (Mouri et al. 1993; Goodrich et al. 1994; Alonso-Herrero et al. 1997; Rodríguez-Ardila et al. 2004, 2005; Riffel et al. 2013).

A 2D diagnostic diagram in the near-infrared was introduced by Larkin et al. (1998) and further developed by Rodríguez-Ardila et al. (2004, 2005) and Riffel et al. (2013). They show that there is a transition from purely ionising radiation (starbursts) to pure supernova-driven shock excitation. Active galactic nuclei are usually located in an intermediate region. Instead of single spectra per galaxy, Colina et al. (2015) use IFS to spatially separate line emitting regions and place them in the diagram. By this method, they can find the typical locations of young star-forming regions, older supernova dominated regions, and the compact AGN dominated region, and show that they occupy different areas in the line-ratio space.

We show the near-infrared diagnostic diagram in Fig. 15. It uses the line ratios $\mathrm{H}_{2} \lambda 2.12 \mu \mathrm{m} / \mathrm{Br} \gamma$ and [Fe II] $\lambda 1.644 \mu \mathrm{m} / \mathrm{Br} \gamma$. The line ratio between the $H$-band [Fe II] and the $K$-band $\mathrm{Br} \gamma$ line is corrected for extinction because at a typical extinction of $A_{V}=5$ the factor is already $\approx 1.6$. However, we did not correct the ratio between $\mathrm{H}_{2} \lambda 2.12 \mu \mathrm{m}$ and $\mathrm{Br} \gamma$ since they are close in wavelength and the correction factor would be only 1.03 . This means the introduced errors due to the uncertainty of the extinction value would be much larger than the actual correction. Therefore, we do not correct line ratios between lines that are both in the $K$ band. 

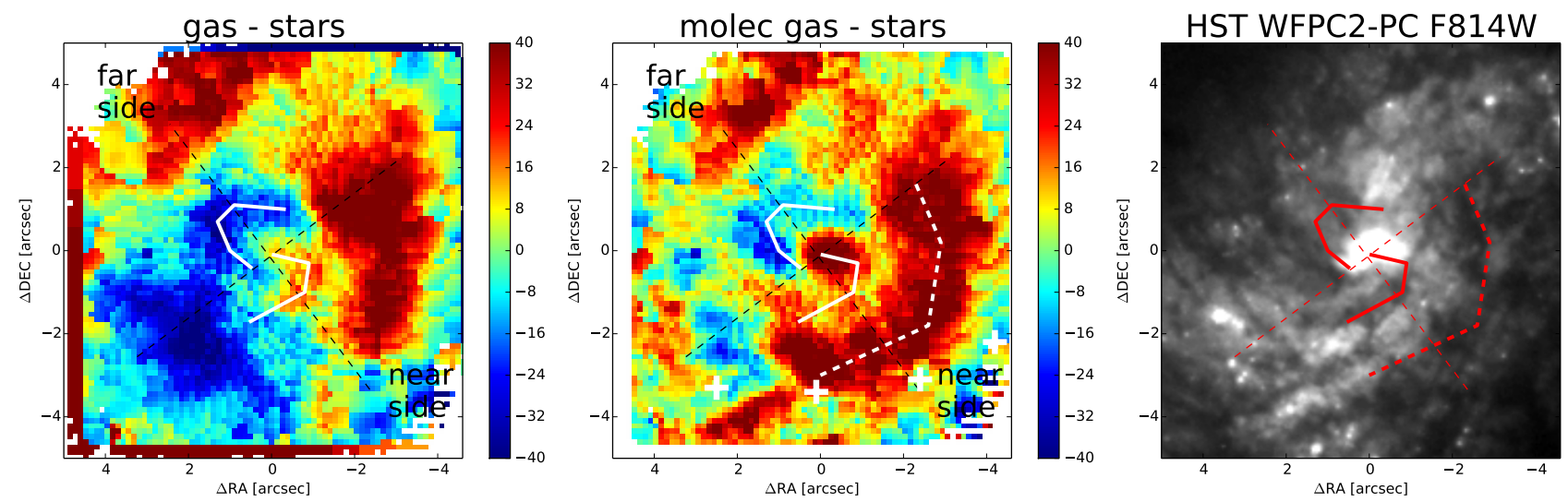

Fig. 14. Residual after subtraction of stellar velocity field from ionised gas (left) and molecular gas (middle). The streaming motion in the southeast coincides with the streaming motion that Salak et al. (2016) observe in CO. To guide the eye, we adopt the white crosses from their Fig. 29 a. Right panel: HST F814W (I-band) map. Comparison of the residual velocities with the optical map shows that possible inflow motions coincide with dust lanes.

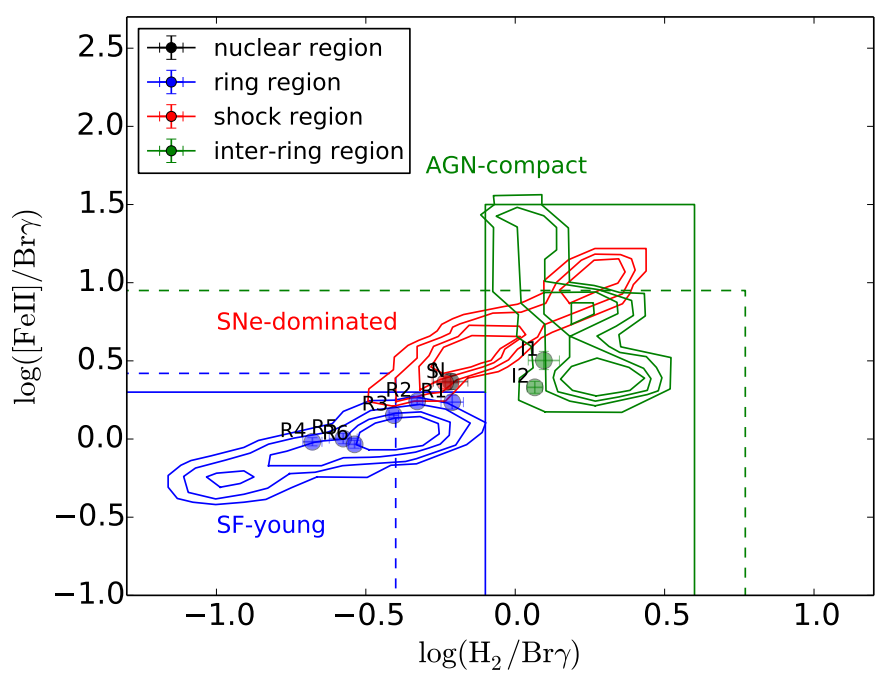

Fig. 15. Near-infrared diagnostic diagram with the line ratios $\log \left(\mathrm{H}_{2} \lambda 2.12 \mu \mathrm{m} / \mathrm{Br} \gamma\right)$ vs. $\log ([\mathrm{Fe} \mathrm{II}] \lambda 1.644 \mu \mathrm{m} / \mathrm{Br} \gamma)$. The contours are from Colina et al. (2015) and denote the regions dominated by young star formation, supernovae, and the compact AGN. Solid lines are upper limits for young star-forming regions and AGN from the same study (based on 2D spectroscopy), while the dashed lines denote the upper limits from 1D spectroscopy studies done by Riffel et al. (2013).

Placing the line ratios of our apertures in the diagram, we first note that all spots in the circumnuclear ring ("R") are located in the region of young star formation. In particular, the spots R4-6 are at even lower ratios than the others, which is an indication of younger starbursts (which emit more $\mathrm{Br} \gamma$ and show fewer supernovae). This is also visible in the line ratio maps in Fig. 7 where these spots clearly form a ring with particularly low values. The other spots have higher line ratios which shifts them into the region of (older) $\mathrm{SNe}$-dominated stellar populations or the compact AGN region. This indicates that their stellar populations are significantly older than those in the circumnuclear ring. Furthermore, their line ratios are typical of AGN. This, however, shows the limitation of the near-infrared diagnostic diagram. There is no confined location for AGN (in contrast to the optical BPT-diagram). Therefore, the position of the spots $\mathrm{S}$, $\mathrm{N}$, and I1-2 in the intermediate region just tells us that they show contributions from both shocks and photoionisation, but not necessarily induced by an AGN.

\subsubsection{Excitation of molecular hydrogen}

Rotational-vibrational transitions are an important cooling channel for molecular gas at temperatures of a few $1000 \mathrm{~K}$. Molecular hydrogen emission lines in the near-infrared are excited by either thermal or non-thermal processes. Thermal processes include the heating of gas by shocks (Hollenbach \& McKee 1989), e.g. due to the interaction of a radio jet with the interstellar medium, or heating by X-rays from the central AGN (Maloney et al. 1996). A non-thermal processes is UV fluorescence (Black \& van Dishoeck 1987) where UV-photons with $912 \AA<\lambda<1500 \AA$ are absorbed by the $\mathrm{H}_{2}$ molecule in the Lyman- and Werner bands, exciting the next two electronic levels $\left(B^{1} \Sigma-X^{1} \Sigma\right.$ and $\left.C^{1} \Pi-X^{1} \Sigma\right)$. With a probability of $90 \%$, a decay into a bound but excited rovibrational level within the electronic ground level $X^{1} \Sigma$ will take place. This mechanism will also populate higher $\mathrm{H}_{2}$ rovibrational levels which are not populated by collisions. Possible sources are OB stars or strong AGN continuum emission.

In theory, all of these excitation processes would produce different $\mathrm{H}_{2}$ spectra such that they could be distinguished by diagnostic line ratios and line population diagrams (Davies et al. 2003; Davies et al. 2005; Rodríguez-Ardila et al. 2004, 2005; Riffel et al. 2013). In practice, however, the different mechanisms usually occur together such that the $\mathrm{H}_{2}$ spectra are mixed (e.g. Zuther et al. 2007; Mazzalay et al. 2013; Busch et al. 2015; Smajić et al. 2015). Even so, $\mathrm{H}_{2}$ line ratios can help estimate the dominating excitation mechanisms or constrain the contributing fractions of different mechanisms. While the $\mathrm{H}_{2} 2-1 \mathrm{~S}(1) / \mathrm{H}_{2}$ 1-0 $\mathrm{S}(1)$ ratio can be used to distinguish between thermal and nonthermal excitation (such as UV fluorescence), the $\mathrm{H}_{2} 1-0 \mathrm{~S}(2) / \mathrm{H}_{2}$ 1-0 $S(0)$ - and also other line ratios between rotational levels in the same vibrational transition, such as $\mathrm{H}_{2}$ 1-0 $\mathrm{S}(3) / \mathrm{H}_{2}$ 1-0S (1)) - can be used to identify thermal UV, shocks, and X-ray excitation.

Furthermore, the rotational excitation temperature can be determined from two ortho/para lines that belong to the same vibrational level, e.g. $\mathrm{H}_{2}$ 1-0 S(0)/ $\mathrm{H}_{2}$ 1-0 S(2), whereas the vibrational excitation temperature can be determined by connecting two transitions with same $J$ but from consecutive $v$ levels, 


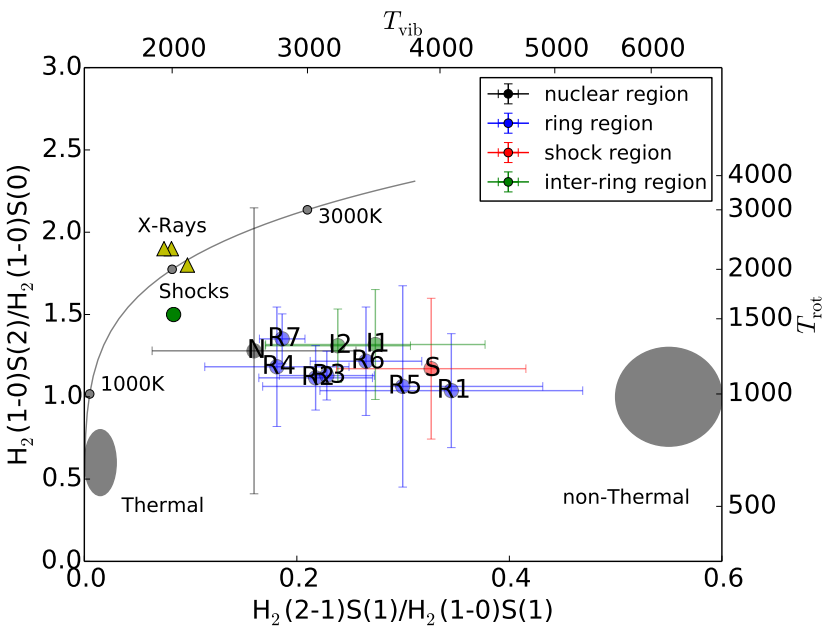

Fig. 16. Molecular hydrogen diagnostic diagram with 2-1S(1)/1-0S(1) vs. 1-0S(2)/1-0S(1) (Mouri 1994). The locations of the apertures are indicated. The location of thermal UV excitation (Sternberg \& Dalgarno 1989) and non-thermal models (Black \& van Dishoeck 1987), as well as the thermal emission curve from $1000 \mathrm{~K}$ to $3000 \mathrm{~K}$ are plotted in grey. The X-ray heating models (Draine \& Woods 1990) are marked by a yellow triangle, the shock-heating model (Brand et al. 1989) by a green circle.

$$
\text { e.g. } \mathrm{H}_{2} \text { 2-1 S(1)/H } 1-0 \mathrm{~S}(1) \text { : }
$$$$
T_{\operatorname{rot}(v=1)}=\frac{1113 \mathrm{~K}}{1.130+\ln \left(\frac{f_{1-0 \mathrm{~S}(0)}}{f_{1-0 \mathrm{~S}(2)}}\right)}
$$$$
T_{\mathrm{vib}}=\frac{5594 \mathrm{~K}}{0.304+\ln \left(\frac{f_{1-0 S(1)}}{f_{2-1 S(1)}}\right)} .
$$

The line ratios of the analysed spots are shown in the $\mathrm{H}_{2}$ excitation diagram in Fig. 16. Furthermore, the vibrational and rotational excitation temperatures of the spots are denoted as upper $x$-axis and right-hand $y$-axis, respectively. We see that the rotational excitation temperature is in the range $1000 \mathrm{~K}<$ $T_{\text {rot }}<1500 \mathrm{~K}$ for all spots. The vibrational temperature, however, reaches from values of $T_{\text {vib }} \approx 2500 \mathrm{~K}$ up to values of $T_{\text {vib }} \lesssim 4000 \mathrm{~K}$. Therefore, we conclude that the spots are not in local thermal equilibrium and a significant contribution of non-thermal fluorescent excitation is very probable. However, we stress that the line ratios do not lie in the region of purely non-thermal excitation, which speaks for a mixture of thermal and non-thermal contribution. The low $\mathrm{H}_{2}$ 1-0 S(2)/ $\mathrm{H}_{2}$ 1-0 S(0) ratios together with rather high values of $\mathrm{H}_{2} 2-1 \mathrm{~S}(1) / \mathrm{H}_{2}$ 1-0 $\mathrm{S}(1)$ (fluorescent excitation) that we find for the spots in the circumnuclear ring are typical for star-forming galaxies. In the near-infrared diagnostic diagram (Sect. 4.3.1, Fig. 15) the spots cannot all be explained by pure shocks, but show a contribution of photoionisation by young stars. This is in particular the case for the spots in the circumnuclear ring. This is consistent with the finding that all spots show significant contribution of nonthermal excitation.

\subsection{Mass of ionised and molecular hydrogen gas}

The mass of hot molecular gas can be estimated from the extinction corrected flux of the $\mathrm{H}_{2} 1-0 \mathrm{~S}(1) \lambda 2.12 \mu \mathrm{m}$ emission line as (e.g. Scoville et al. 1982)

$M_{\mathrm{H}_{2}}=\frac{2 m_{\mathrm{p}} F_{1-0 \mathrm{~S}(1)} 4 \pi D_{L}^{2}}{f_{v=1, J=3} A_{1-0 \mathrm{~S}(1)} h c / \lambda}$, where $D_{L}$ is the luminosity distance of the galaxy and $m_{\mathrm{p}}$ the proton mass. Furthermore, we need the population fraction of the upper energy level $f_{v=1, J=3}$ and the Einstein coefficient $A_{1-0 \mathrm{~S}(1)}=$ $3.47 \times 10^{-7} \mathrm{~s}^{-1}$ (Turner et al. 1977), which is the probability that a particular transition from this level takes place. The population fraction depends strongly on the vibrational temperature of the system, with a dependency $f_{v, J} \propto g_{J} \times \exp \left(E_{v, J} / k_{B} T\right)$. Most authors assume (1) a local thermal equilibrium (LTE, from which follows that the level population is only determined by the system temperature) and (2) a typical vibrational temperature of $T_{\text {vib }}=2000 \mathrm{~K}$. For this temperature the population fraction is $f_{v=1, J=3}=1.22 \times 10^{-2}$. For better comparison, we use this value and find the following relation between warm molecular gas mass and extinction corrected emission line flux $F_{1-0 S(1)}$,

$M_{\mathrm{H}_{2}}=5.0776 \times 10^{16}\left(\frac{D_{L}}{\mathrm{Mpc}}\right)^{2}\left(\frac{F_{1-0 \mathrm{~S}(1)}}{\mathrm{W} \mathrm{m}^{-2}}\right) M_{\odot}$,

where $D_{L}$ is the distance to the galaxy in Mpc (Scoville et al. 1982; Wolniewicz et al. 1998; Riffel et al. 2010).

As indicated before, the assumption of LTE might not always be justified. Furthermore, we see indications of a higher vibrational temperature. Therefore, we calculate the population fraction

$f_{v=1, J=3}=\frac{g_{J=3} \times \exp \left(E_{v=1, J=3} / k_{B} T_{\mathrm{vib}}\right)}{\sum_{v, J} g_{J} \times \exp \left(E_{v, J} / k_{B} T\right)}$

for higher temperatures as well. We find that $f(T=$ $3000 \mathrm{~K}) / f(T=2000 \mathrm{~K}) \approx 1.8$ and $f(T=4000 \mathrm{~K}) / f(T=$ $2000 \mathrm{~K}) \approx 2.0$. This means that for temperatures higher than $T=2000 \mathrm{~K}$, we would overestimate the gas mass.

In general, the hot molecular gas (as traced by the nearinfrared $\mathrm{H}_{2}$ emission lines) only represents the hot surface, and therefore a small fraction, of the total gas amount of the galaxy. A further difficulty is that the strength of the emission line does not only depend on the gas mass but also on the external energy source that is able to excite the rovibrational transitions (e.g. UV-photons from OB stars or shocks induced by supernovae or AGN outflows). Nevertheless, empirical relations between the near-infrared $\mathrm{H}_{2}$ luminosities and $\mathrm{CO}$ luminosities (which are common tracers of the total molecular gas mass) suggest that the luminosity of near-infrared $\mathrm{H}_{2}$ lines can be used to estimate the cold gas mass. We use the conversion factor $M_{\mathrm{H}_{2} \text { (cold) }} / M_{\mathrm{H}_{2}(\text { warm })}=(0.3-1.6) \times 10^{6}$ from Mazzalay et al. (2013). Other consistent cold-to-warm $\mathrm{H}_{2}$ gas mass conversion factors are $10^{5}-10^{7}$ (Dale et al. 2005) and $2.5 \times 10^{6}$ (with a $1 \sigma$ uncertainty of a factor of 2; Müller Sánchez et al. 2006).

The mass of ionised hydrogen gas can be estimated from the measured and extinction corrected flux of hydrogen recombination lines such as $\mathrm{Pa} \alpha$ or $\mathrm{Br} \gamma$. Assuming an electron temperature of $T=10^{4} \mathrm{~K}$ and a density in the range $10^{2}<n_{\mathrm{e}}<10^{4} \mathrm{~cm}^{-3}$, we can calculate the ionised gas mass from the extinction corrected $\mathrm{Pa} \alpha$ and $\mathrm{Br} \gamma$ fluxes, $f_{\mathrm{Pa} \alpha}$ and $f_{\mathrm{Br} \gamma}$ by

$$
\begin{aligned}
M_{\mathrm{H} \mathrm{II}} & \approx 2.4 \times 10^{21}\left(\frac{f_{\mathrm{Pa} \alpha}}{\mathrm{W} \mathrm{m}^{-2}}\right)\left(\frac{D}{\mathrm{Mpc}}\right)^{2}\left(\frac{n_{\mathrm{e}}}{\mathrm{cm}^{-3}}\right)^{-1} M_{\odot} \\
& \approx 2.9 \times 10^{22}\left(\frac{f_{\mathrm{Br} \gamma}}{\mathrm{W} \mathrm{m}^{-2}}\right)\left(\frac{D}{\mathrm{Mpc}}\right)^{2}\left(\frac{n_{\mathrm{e}}}{\mathrm{cm}^{-3}}\right)^{-1} M_{\odot}
\end{aligned}
$$

where $D$ is the luminosity distance to the galaxy and $n_{\mathrm{e}}$ the electron density (see Busch et al. 2015, for details). Here, we use typical values of $T=10^{4} \mathrm{~K}$ and $n_{\mathrm{e}}=10^{2} \mathrm{~cm}^{-2}$. 
Given the mentioned systematic uncertainties and difficulties with the gas mass estimates that are much higher than those arising from (emission line flux) measurements, we refrain from stating formal errors when calculating gas masses, but point out that these are only order-of-magnitude estimates.

We estimate the gas masses by summing over the FOV $\left(\sim 10^{\prime \prime} \times 10^{\prime \prime}\right)$. We estimate the average extinction in this FOV to be $A_{V} \approx 2 \mathrm{mag}$. Applying this correction and using the $\mathrm{H}_{2} \lambda 2.12 \mu \mathrm{m}$ emission line, we estimate a hot molecular gas mass of $\sim 730 M_{\odot}$, which corresponds to a cold molecular gas mass of $\sim(2-12) \times 10^{8} M_{\odot}$. This is in good agreement with the molecular gas mass of $4.2 \times 10^{8} M_{\odot}$ that Salak et al. (2014) derive from CO-measurements with ASTE and $2 \times 10^{8} M_{\odot}$ that Salak et al. (2016) derive from ALMA CO(1-0) measurements (in an aperture of $<250 \mathrm{pc}$ corresponding to our FOV; however, it is a lower limit as it is not corrected for missing short baselines) ${ }^{6}$. Using the $K$-band emission line $\mathrm{Br} \gamma$, we estimate the ionised hydrogen mass $M_{\mathrm{H} \text { II }} \sim 6.6 \times 10^{6} M_{\odot}$. We see that the ionised hydrogen gas mass is $\sim 9000$ times higher than the hot molecular gas mass. This is in agreement with typical ratios of $10^{3}-10^{4}$ (Riffel et al. 2014, and references therein). For the nuclear regions of nearby Seyfert galaxies, the AGNIFS group obtained hot molecular gas masses with a range of $10^{1}<M_{\mathrm{H}_{2}}<10^{3} M_{\odot}$ and ionised gas masses with a range of $10^{4}<M_{\mathrm{H}}$ II $<10^{7} M_{\odot}$ (Riffel et al. 2015b, and references therein). For NUGA sources, cold molecular gas masses (derived from CO-emission) range from $10^{8}-10^{10} M_{\odot}$, with typical masses of the order of several $10^{9} M_{\odot}$ (Moser et al. 2012, and references therein), whereas lowluminosity QSOs at higher redshift $(0.02 \leq z \leq 0.06)$ have systematically higher gas reservoirs (Busch et al. 2015; Moser et al. 2016). The nuclear region of NGC1808 lies in the first range and therefore shows a typical behaviour for local Seyfert galaxies. However, we stress that the derived gas masses can only be used as order-of-magnitude estimates and only the combination of near-infrared observations together with high-resolution sub-mm interferometry (for example with ALMA) can provide a full view of the galactic centres and their warm and cold gas reservoirs.

We also measure the gas masses in the apertures. The masses are listed in Tables 1 and 2. In addition, we also list the gas mass surface densities of the cold molecular gas. These densities will be compared with the star formation surface densities in Sect. 4.5.1 to determine the efficiency of the formation of new stars out of the available amount of gas.

\subsection{Star formation properties}

One of the most striking features in the central region of NGC 1808 is the patchy circumnuclear ring that is commonly associated with a star-forming ring (e.g. Krabbe et al. 1994; Kotilainen et al. 1996; Tacconi-Garman et al. 1996). The positions in the diagnostic diagrams strongly support this. In the near-infrared diagnostic diagram (Fig. 15) the spots in the ring show very low line ratios, indicating pure photoionisation which is indicative of star formation. In the $\mathrm{H}_{2}$ excitation diagram (Fig. 16), the spots show a significant contribution of nonthermal excitation, for example by UV fluorescence which is consistent with star-forming clumps. Furthermore, we find a significantly lower stellar velocity dispersion in the region of the ring, which is interpreted as being due to the presence of younger stellar populations that have formed out of cooled molecular gas and thus not dispersed into the surrounding region (Sect. 4.1).

\footnotetext{
6 We translated their measurements of $3.0 \times 10^{8} M_{\odot}$ and $1.4 \times 10^{8} M_{\odot}$ at a luminosity distance of $10.8 \mathrm{Mpc}$ to our assumed distance of $12.8 \mathrm{Mpc}$.
}

\subsubsection{Star formation rates and efficiency}

In starburst events, a large number of stars are formed, some of them hot and luminous $\mathrm{O}$ and B stars. These immediately start to photoionise the surrounding interstellar medium. This produces large nebular regions which can show strong nebular emission like the hydrogen recombination lines $\mathrm{H} \alpha, \mathrm{H} \beta$ (in the optical), $\mathrm{Pa} \alpha$, or $\mathrm{Br} \gamma$ (in the near-infrared). The UV photons are also absorbed by dust, which is heated to $30-60 \mathrm{~K}$ and then reradiates the energy at wavelengths of around $60 \mu \mathrm{m}$.

The star formation rate is a good estimator of the power of the starburst. It can be calculated from the luminosity of hydrogen recombination lines since they are proportional to the Lyman continuum which is proportional to the star formation rate (SFR). Independent of the star formation history, only stars with masses $\gtrsim 10 M_{\odot}$ and ages $\lesssim 20$ Myr contribute to the ionising flux. Therefore, hydrogen recombination lines trace the instantaneous SFR (Kennicutt 1998a). We derive the SFR with the calibration of Panuzzo et al. $(2003)^{7}$ :

$$
S F R_{\mathrm{Br} \gamma}=\frac{L(\mathrm{Br} \gamma)}{1.585 \times 10^{32} \mathrm{~W}} M_{\odot} \mathrm{yr}^{-1}
$$

In the apertures that are located in the star-forming ring the star formation rates range from 0.04 to $0.09 M_{\odot} \mathrm{yr}^{-1}$. In the nuclear aperture, we find a star formation rate of $\sim 0.18 M_{\odot} \mathrm{yr}^{-1}$. All measurements are listed in Table 3. Esquej et al. (2014) estimate the nuclear star formation rate from the $11.3 \mu \mathrm{m}$ emission of Polycyclic Aromatic Hydrocarbons (PAH). In a slit with 0'.35 width, they measure $0.21 M_{\odot} \mathrm{yr}^{-1}$. This is higher than the value that we measure in our larger $00^{\prime \prime} .75$ nuclear aperture. However, the PAH feature traces star formation integrated over a few tens of Myr, while we use hydrogen recombination lines to estimate the instantaneous star formation rate. With this in mind, the star formation rate estimates are consistent.

To better compare the values with other measurements, we divide by the area of the apertures, which have a radius of $0.75 \approx 50 \mathrm{pc}$. The star formation surface density then ranges from 5 to $13 M_{\odot} \mathrm{yr}^{-1} \mathrm{kpc}^{-2}$ in the ring and is $\sim 28 M_{\odot} \mathrm{yr}^{-1} \mathrm{kpc}^{-2}$ in the nucleus. Typically, star formation surface density is in the range $(1-50) M_{\odot} \mathrm{yr}^{-1} \mathrm{kpc}^{-2}$ on hundreds of parsec scales, $(50-500) M_{\odot} \mathrm{yr}^{-1} \mathrm{kpc}^{-2}$ on scales of tens of parsecs, and increases up to some $1000 M_{\odot} \mathrm{yr}^{-1} \mathrm{kpc}^{-2}$ on parsec scales (Valencia-S. et al. 2012, and references therein). We conclude that the star formation rates in the apertures are of a typical order of magnitude.

Another question is how efficient star formation is, i.e. the transformation of gas into young stars. For this, we evaluate the location of our data in the Kennicutt-Schmidt law, which states that the star formation surface density scales with the local gas surface density as $\Sigma_{\mathrm{SFR}} \propto\left(\Sigma_{\text {gas }}\right)^{n}$ (Schmidt 1959; Kennicutt 1998b; Bigiel et al. 2008). In Fig. 17 we show the data points collected by Bigiel et al. (2008) together with the locations of our apertures. We see that the spots in the ring that show high star formation rates are all in the Kennicutt-Schmidt relation. Some of them (particularly spots R3-R6 with high SFR) are at

\footnotetext{
7 While the uncertainty on the SFR introduced by line measurement errors can be estimated in a relatively straightforward manner, there are further uncertainties coming from the luminosity distance/cosmology, the extinction correction, and most importantly the calibration of the SF-estimator. As a conservative estimate we have to assume an uncertainty of at least a factor of two. Therefore, we do not state particular uncertainties of the derived SFR values, but we note that they should be considered more as an order-of-magnitude estimate. The same is valid for the supernova rate that we estimate in the next section.
} 


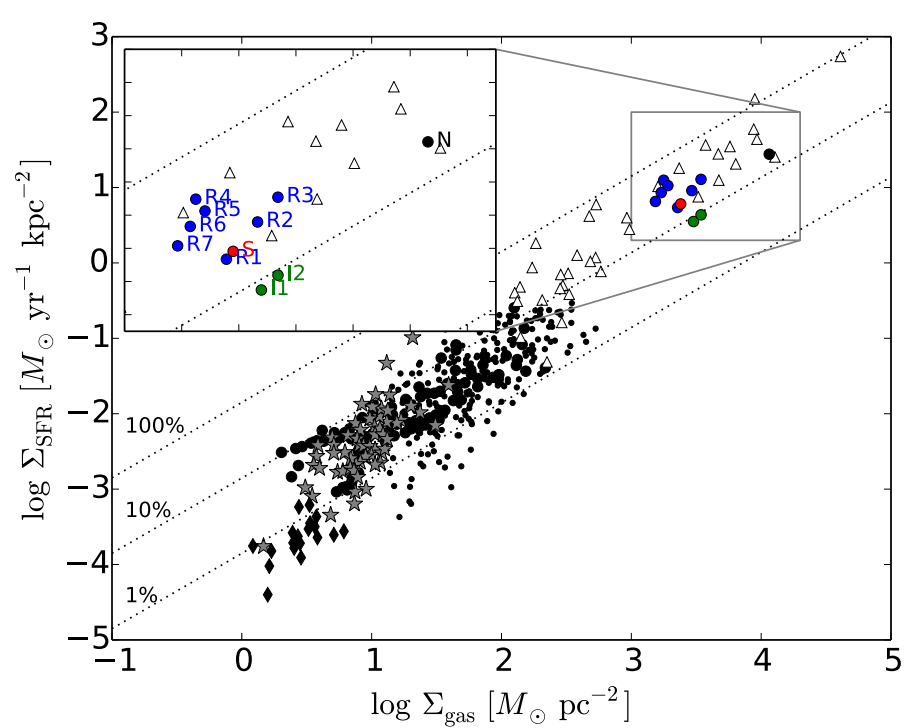

Fig. 17. Kennicutt-Schmidt relation of star formation: relation between the gas surface density, $\Sigma_{\text {gas }}$, and the star formation surface density, $\Sigma_{\mathrm{SFR}}$. The black/grey data points are from Bigiel et al. (2008). Our measurements for NGC 1808 are indicated by big black (nucleus), blue (star formation ring), red (region $S$ ), and green (regions between ring and nucleus) circles.

the upper end, which indicates high star formation efficiency. Apertures R1, S, and nuclear aperture $\mathrm{N}$ are located at the lower part of the relation, which indicates less efficient star formation. Apertures I1 and I2, between the ring and the nucleus, show even lower star formation efficiencies, which indicates that they do not host starbursts; instead, the starbursts are confined in the star formation ring and the nuclear starburst.

\subsubsection{Star formation in the circumnuclear ring}

While it is clear that a circumnuclear star-forming ring provides the necessary gas reservoir for star formation, a further question is the sequence in which star formation is taking place. Böker et al. (2008) suggest two scenarios which they call "popcorn" and "Pearls on a string". In the first scenario, gravitational instabilities fragment the rings in the inner Lindblad resonance into several clouds. Starbursts then occur whenever gas accretion leads to a critical density. The location of these starbursts is fully stochastic and, therefore, no age gradient is seen (Elmegreen 1994). In the second scenario, star formation only occurs in particular "overdensity regions" (usually two, often located close to the point where the gas enters the ring). Then the young clusters move along the ring, following the gas movement, and meanwhile age, resulting in an age gradient along the ring (e.g. Falcón-Barroso et al. 2014; Väisänen et al. 2014).

While it is difficult to determine the absolute age of the young clusters, Böker et al. (2008) suggest a method for at least determining the relative age of the clusters in order to identify possible age gradients. Following their method, we created a false-colour map that shows the emission of He I in blue, Br $\gamma$ in green, and [Fe II] in red. The idea is that since He I has a higher ionisation potential than $\mathrm{Br} \gamma$, it requires hotter and more massive stars than $\mathrm{Br} \gamma$. He I will therefore only be visible in the very early phase of an instantaneous starburst. On the other hand [Fe II] traces supernovae, which become dominant after $\sim 10 \mathrm{Myr}$ when the most massive stars arrive at the end of their lifetimes. Based on this argumentation, we can now identify an age sequence in

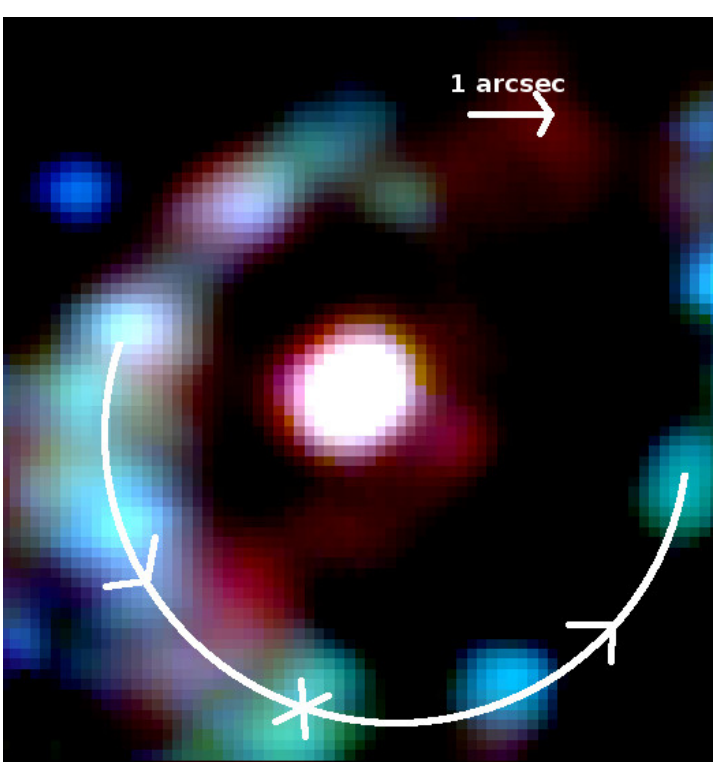

Fig. 18. False-colour map of the emission line fluxes in NGC 1808 following the method described in Böker et al. (2008). The [Fe II] emission is red, $\mathrm{Br} \gamma$ green, and He I blue. The youngest regions appear in blue, the oldest in red. The direction of the rotation is indicated by arrows, the overdensity region where a possible gas spiral hits the ring by a cross. The centre is red, indicating that it is dominated by supernova remnants as traced by [Fe II] emission. In the ring, no clear age gradient is visible from the false-colour map.

the false-colour map, where blue traces the youngest clusters, green intermediate age clusters, and red the oldest clusters. In Fig. 18, we can indeed see that the clusters have different relative ages. In particular, the clusters in the south-east and southwest, which correspond to the apertures R4 and R6, show rather low ages, consistent with the near-infrared diagnostic diagrams (Figs. 15 and 16). The central aperture, however, is dominated by supernova remnants. This is in agreement with previous age estimates of the nuclear starburst that hint at an age of $\gtrsim 10^{7} \mathrm{Myr}$ (Krabbe et al. 1994; Kotilainen et al. 1996). Based on our data, we do not find evidence of an age gradient as predicted by the "Pearl on a string" scenario. A more likely scenario is that the clusters form at random positions in the circumnuclear gas ring and then drift away following the more circular orbits of the stellar velocity field (Galliano \& Alloin 2008; Galliano et al. 2014).

In the following, we want to get an estimate of the absolute age of the starbursts. We use STARBURST99 (Leitherer et al. 1999, 2010, 2014; Vázquez \& Leitherer 2005) to predict parameters such as the equivalent width of $\mathrm{Br} \gamma$ for different star formation histories ${ }^{8}$. These models can then be compared to observations to determine the age of the circumnuclear star formation regions and constrain the star formation history (e.g. Davies et al. 2006, 2007; Dors et al. 2008; Riffel et al. 2009; Levesque \& Leitherer 2013; Busch et al. 2015). In Fig. 19, we show simulations of the equivalent width of $\operatorname{Br} \gamma$ and the supernova rate normalised by the $K$-band continuum luminosity.

[Fe II] is a coolant in supernova remnants, but is absent in pure HII regions. Therefore, under the assumption that [Fe II] is primarily excited by shocks caused by supernova explosions, the supernova rate can be estimated from the [Fe II] luminosity. Forbes et al. (1992) argue that the radio hot spots in NGC 1808

8 We use an instantaneous starburst model with total stellar mass $10^{6} M_{\odot}$, a Kroupa IMF with two IMF intervals with $\alpha=1.3$ for the mass interval $0.1<m<0.5$ and $\alpha=2.3$ for $0.5<m<100$, and Geneva tracks, including AGB stars. 
Table 3. Star formation rate (SFR) and SFR density, Bry equivalent width, supernova rate (SNR) and SNR normalised by $K$-band continuum luminosity for all apertures.

\begin{tabular}{cccccc}
\hline \hline Aperture & $\begin{array}{c}S F R_{\mathrm{Br} \gamma} \\
{\left[10^{-3} M_{\odot} \mathrm{yr}^{-1}\right]}\end{array}$ & $\begin{array}{c}\log \left(\Sigma_{\mathrm{SFR}}\right) \\
{\left[M_{\odot} \mathrm{yr}^{-1} \mathrm{kpc}^{-1}\right]}\end{array}$ & $\begin{array}{c}W_{\mathrm{Br} \gamma} \\
{[\AA \AA]}\end{array}$ & $\begin{array}{c}S N R \\
{\left[10^{-5} \mathrm{yr}^{-1}\right]}\end{array}$ & $\begin{array}{c}S N R / L_{K} \\
{\left[\mathrm{yr}^{-1} / 10^{12} L_{\odot, K}\right]}\end{array}$ \\
\hline $\mathrm{N}$ & 188 & 1.44 & $4.5 \pm 0.3$ & 561 & 2.5 \\
$\mathrm{R} 1$ & 37 & 0.73 & $3.9 \pm 0.2$ & 81 & 1.4 \\
$\mathrm{R} 2$ & 62 & 0.96 & $9.9 \pm 0.2$ & 138 & 3.9 \\
$\mathrm{R} 3$ & 87 & 1.11 & $14.7 \pm 0.2$ & 159 & 4.7 \\
$\mathrm{R} 4$ & 85 & 1.10 & $13.3 \pm 0.2$ & 104 & 2.6 \\
$\mathrm{R} 5$ & 72 & 1.03 & $9.1 \pm 0.2$ & 93 & 1.9 \\
$\mathrm{R} 6$ & 58 & 0.93 & $14.7 \pm 0.2$ & 69 & 3.1 \\
$\mathrm{R} 7$ & $44^{a}$ & $0.82^{a}$ & $18.0 \pm 0.2$ & $29^{a}$ & 1.6 \\
$\mathrm{~S}$ & 41 & 0.78 & $4.3 \pm 0.2$ & 121 & 2.0 \\
$\mathrm{I} 1$ & 24 & 0.55 & $1.9 \pm 0.2$ & 98 & 1.4 \\
$\mathrm{I} 2$ & 30 & 0.64 & $3.9 \pm 0.2$ & 81 & 1.8 \\
\hline
\end{tabular}

Notes. All apertures have a radius of 0 ' 75 which corresponds to a physical area of $6800 \mathrm{pc}^{2} .{ }^{(a)}$ Values are not corrected for extinction because extinction could not be determined in this aperture.
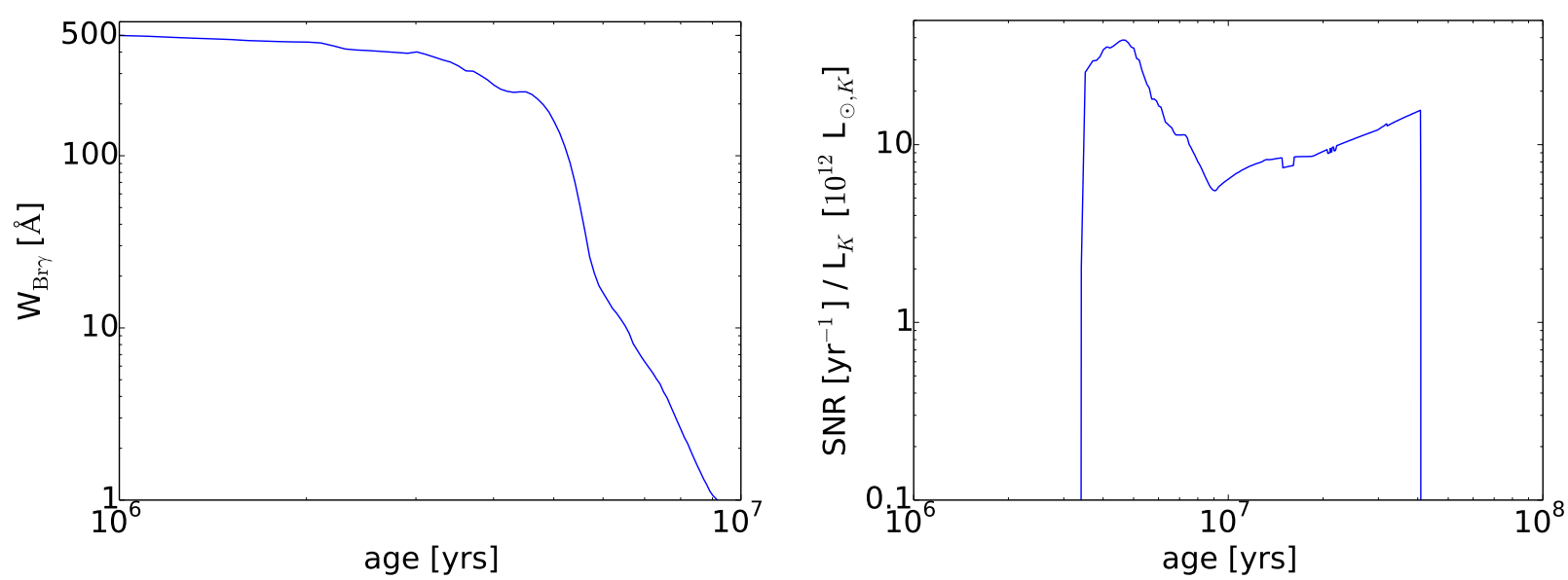

Fig. 19. Equivalent width of $\operatorname{Br} \gamma(l e f t)$ and supernova rate (normalised by $K$-band luminosity, right) as predicted by STARBURST99.

can be explained by supernova remnants (SNRs) alone. In Fig. 4, we show the $[\mathrm{Fe}$ II] emission together with the radio contours. They coincide well, which supports the idea that [Fe II] and radio emission are indeed both produced by the same mechanism, probably shocks due to supernovae. Furthermore, the radio spots coincide with mid-infrared hot spots, which indicates that they are not isolated SNRs but are associated with young star clusters (Galliano et al. 2005). We thus use the [Fe II] emission to estimate the supernova rate in the apertures, using the calibration of Alonso-Herrero et al. (2003):

$S N R=8.08 \frac{L_{[\mathrm{Fe} \mathrm{II}]}}{10^{35} \mathrm{~W}} \mathrm{yr}^{-1}$.

Since both observables, the Bry equivalent width and the supernova rate from [Fe II], have to be normalised by the continuum luminosity, a proper estimation of the contribution due to the underlying old bulge population is crucial. Riffel et al. (2009) perform aperture photometry in the star formation regions and in the neighbouring regions and find that the bulge contributes $\sim 50 \%$ to the total flux of the star formation regions. We lay slits through the star formation spots and inspect the light profiles. In a first-order approximation, we then subtract a linear function from the profiles to distinguish between the smooth distribution of the underlying old population and the additional contribution of the young stellar clusters to the continuum flux in the apertures. In spots R2, R3, and R4, we find bulge contributions of $75 \%, 80 \%$, and $70 \%$, respectively. The observed equivalent widths must therefore be increased by factors of 4,5 , and 3.3 , respectively. In the other apertures, the contribution of the old population is $\gtrsim 90 \%$. Thus, we have to keep in mind that the equivalent widths might be underestimated by a factor of at least ten.

To estimate the age of the starbursts in R2, R3, and R4, we correct the Br $\gamma$ equivalent width and normalised SNR in Table 3 and compare them to the STARBURST99 predictions in Fig. 19. Assuming an instantaneous starburst, the favoured models give starburst ages of the order of $6 \mathrm{Myr}$ (R2), $5 \mathrm{Myr}$ (R3), and 6-8 Myr (R4). This is in good agreement with the estimates from near-infrared data, which result in an age range between 5 and 20 Myr (Krabbe et al. 1994; Tacconi-Garman et al. 1996; Kotilainen et al. 1996) and from mid-infrared data which result in an age range between 3 and 6 Myr (Galliano et al. 2005).

The physical rotational velocity of the ring can be calculated from the observed velocity $v_{\text {obs }}$ at an angle $\theta$ in the plane of the ring by

$v_{\text {ring }}=\frac{v_{\text {obs }}}{\sin (i) \cos (\theta)}$,

where $i$ is the inclination of $52^{\circ}$. From the Br $\gamma$ LOSV map (Fig. 10), we estimate the rotation velocity to be 
$v_{\text {ring }} \approx 170-180 \mathrm{~km} \mathrm{~s}^{-1}$. With this velocity and the ring radius of $r \approx 190 \mathrm{pc}$ at hand, we can calculate the time needed for a cloud in the ring to complete one orbit:

$t_{\text {travel }}=\frac{2 \pi \times 190 \mathrm{pc}}{170 \mathrm{~km} \mathrm{~s}^{-1}} \approx 7 \mathrm{Myr}$

Comparing the star cluster ages derived above with the travel time, we conclude that the clusters have travelled almost one complete orbit since formation. This is similar to the case of NGC 7552 (Brandl et al. 2012).

Simulations by Seo \& Kim (2013) show that the star formation rate could be decisive for the way star formation happens in circumnuclear rings. When the SFR is low, star formation mostly takes place in the contact points of the ring with the dust lanes, leading to an age gradient in the ring (Pearls on a string), while for high SFR, star formation is randomly distributed in the ring (popcorn). They find a critical SFR of $\sim 1 M_{\odot} \mathrm{yr}^{-1}$. This is supported by a $\mathrm{H} \alpha$ study of 22 circumnuclear rings (Mazzuca et al. 2008). However, these authors find a higher critical SFR of $\sim 3 M_{\odot} \mathrm{yr}^{-1}$ and a large spread in the SFR. By summing up the SFRs in the different apertures, we realise that the circumnuclear SFR in NGC 1808 is of the order of $1 M_{\odot} \mathrm{yr}^{-1}$, which is the suspected transition value between the two models.

\subsection{Nuclear activity: Does NGC1808 host an AGN?}

Many authors have followed the old classification of NGC1808 as an AGN by Veron-Cetty \& Veron (1985) which was based on the detection of two line systems of different widths. The one with the broader emission lines showed a flux ratio $\mathrm{H} \alpha /[\mathrm{N} \mathrm{II}]=$ $0.94 \pm 0.05$ which is typical for Seyfert galaxies. More recent optical spectroscopy is available from the S7 survey (Dopita et al. 2015), where the authors measure line ratios of $\log ([\mathrm{O} \mathrm{III}] / \mathrm{H} \beta)=$ $-0.58, \log ([\mathrm{N} \mathrm{II}] / \mathrm{H} \alpha)=-0.08$, and $\log ([\mathrm{S} \mathrm{II}] / \mathrm{H} \alpha)=-0.59$ which are typical line ratios of $\mathrm{H}$ II regions. In accordance with this, several other authors do not find evidence to justify the old classification as a Seyfert-galaxy and state that the central activity can be explained by intense star formation alone which comes along with strong supernova remnants (e.g. Forbes et al. 1992) and a superwind (e.g. Phillips 1993). Krabbe et al. (2001) establish a mid-infrared X-ray correlation and argue that the position of NGC 1808 in this diagram clearly indicates a pure starburst.

We analyse our emission line measurements in the nearinfrared diagnostic diagram in Fig. 15 and show that the nuclear aperture has line ratios typical for supernova dominated regions. The line ratios are close to the region of young star formation from which we conclude that there is no evidence of strong AGN activity and the nucleus shows characteristics of an aging starburst.

NGC 1808 was detected by XMM with a hard X-ray luminosity of $L_{\text {hardX-ray }}=10^{40.4} \mathrm{erg} \mathrm{s}^{-1}$. If we supposed that the X-ray flux was produced by an AGN, we could calculate the AGN luminosity, resulting in $L(\mathrm{AGN})=10^{41.2} \mathrm{erg} \mathrm{s}^{-1}$ (Brightman \& Nandra 2011; Esquej et al. 2014). The Eddington ratio is defined by $\lambda \equiv L(\mathrm{AGN}) / L_{\mathrm{Edd}}$ with Eddington luminosity $L_{\mathrm{Edd}}=1.26 \times 10^{38}\left(M_{\mathrm{BH}} / M_{\odot}\right) \mathrm{erg} \mathrm{s}^{-1}$. The black hole mass is of the order of $M_{\mathrm{BH}} \approx 5 \times 10^{7} M_{\odot}$ (Sect. 4.1). With these values, the Eddington ratio is $\log (\lambda)=-4.4$, which is the Eddington ratio of a low-luminosity AGN. We conclude that an AGN cannot be fully ruled out. However, if present it would be very weak. The dominating excitation mechanism in the centre is star formation and associated supernovae.

\section{Conclusions and summary}

We have analysed near-infrared IFS of the central kiloparsec of the galaxy NGC 1808 with the following results:

- We study the star formation history of the hotspots in the circumnuclear ring. We determine the ages to be $\lesssim 10 \mathrm{Myr}$. We also study the relative ages. We cannot find evidence of an age gradient that would be predicted by the "Pearls on a string" model. However, the travel time around the ring is comparable to the cluster ages, which means that the clusters have already travelled around a significant portion of the ring.

- In a FOV of $10^{\prime \prime} \times 10^{\prime \prime}$ (which corresponds to $\sim 600 \mathrm{pc}$ ), we measure the $\mathrm{H}_{2} \lambda 2.12 \mu \mathrm{m}$ line emission to estimate the molecular gas mass. For the hot molecular gas, we find a mass of $\sim 730 M_{\odot}$, which corresponds to a cold molecular gas mass of $(2-12) \times 10^{8} M_{\odot}$. From the $\mathrm{Br} \gamma$ line, we estimate an ionised gas mass of $\sim 6.6 \times 10^{6} M_{\odot}$. All gas masses are typical for NUGA sources.

- From the $M_{\mathrm{BH}}-\sigma_{*}$ relation, we calculate a black hole mass of $\sim(1-5) \times 10^{7} M_{\odot}$. Since NGC 1808 shows the signatures of a pseudo-bulge, this should be interpreted as a rough estimate only.

- We fit the CO absorption band heads in the $K$-band to obtain the stellar velocity field. It shows an overall rotation. However an S-shaped zero-velocity line, a drop in the central velocity dispersion, and residual structures after subtracting a pure rotation model indicate the presence of a bar and probably a nuclear disc.

- The gaseous kinematics also shows an overall rotation. However, the deviations from pure rotation are much greater than in the stellar velocity field. By subtracting the stellar velocity field from the gas velocity field, we find a residual structure in the central $\sim 100 \mathrm{pc}$ that has the shape of a twoarm nuclear spiral and could indicate an inflowing streaming motion.

To summarise, we find a large gas reservoir and a disturbed gas velocity field that even shows signs of inflowing motion to the inner tens of parsecs. However, we do not find indications of strong AGN activity (if AGN is present, its Eddington ratio is as low as $\log (\eta) \lesssim-4.4)$. Instead, the gas seems to be used up for star formation that is occurring in the circumnuclear ring and in the nuclear starburst. This shows that for an AGN to be present, it is not enough to have gas and drive it to the centre. Furthermore, it raises the questions: What decides whether gas is used for AGN fuelling or star formation? Is there any sufficient condition for AGN activity?

Upcoming ALMA observations with higher angular resolution will trace the cold gas mass and structure and will show whether they show similar inflowing streaming motions like the warm gas.

Acknowledgements. The authors thank Florian Peißker and the team of the Paranal Observatory who conducted the SINFONI observations. Furthermore, we thank the anonymous referee for comments that helped to clarify the manuscript. This work was supported by the Deutsche Forschungsgemeinschaft (DFG) via SFB 956, subproject A2. G. Busch and N. Fazeli are members of the Bonn-Cologne Graduate School of Physics and Astronomy (BCGS). M. Valencia-S. received funding from the European Union Seventh Framework Programme (FP7/2007-2013) under grant agreement No. 312789. Based on observations made with the NASA/ESA Hubble Space Telescope, and obtained from the Hubble Legacy Archive, which is a collaboration between the Space Telescope Science Institute (STScI/NASA), the Space Telescope European Coordinating Facility (ST-ECF/ESA) and the Canadian Astronomy Data Centre (CADC/NRC/CSA). 


\section{References}

Alonso-Herrero, A., Rieke, M. J., Rieke, G. H., \& Ruiz, M. 1997, ApJ, 482, 747 Alonso-Herrero, A., Rieke, G. H., Rieke, M. J., \& Kelly, D. M. 2003, AJ, 125, 1210

Baldwin, J. A., Phillips, M. M., \& Terlevich, R. 1981, PASP, 93, 5

Barbosa, F. K. B., Storchi-Bergmann, T., Cid Fernandes, R., Winge, C., \& Schmitt, H. 2006, MNRAS, 371, 170

Bedregal, A. G., Colina, L., Alonso-Herrero, A., \& Arribas, S. 2009, ApJ, 698 1852

Bigiel, F., Leroy, A., Walter, F., et al. 2008, AJ, 136, 2846

Black, J. H., \& van Dishoeck, E. F. 1987, ApJ, 322, 412

Böker, T., Falcón-Barroso, J., Schinnerer, E., Knapen, J. H., \& Ryder, S. 2008, AJ, 135, 479

Bonnet, H., Abuter, R., Baker, A., et al. 2004, The Messenger, 117, 17

Brand, P. W. J. L., Toner, M. P., Geballe, T. R., et al. 1989, MNRAS, 236, 929

Brandl, B. R., Martín-Hernández, N. L., Schaerer, D., Rosenberg, M., \& van der Werf, P. P. 2012, A\&A, 543, A61

Bremer, M., Scharwächter, J., Eckart, A., et al. 2013, A\&A, 558, A34

Brightman, M., \& Nandra, K. 2011, MNRAS, 413, 1206

Busch, G., Fazeli, N., Eckart, A., et al. 2016, A\&A, 587, A138

Busch, G., Smajić, S., Scharwächter, J., et al. 2015, A\&A, 575, A128

Calzetti, D., Armus, L., Bohlin, R. C., et al. 2000, ApJ, 533, 682

Cappellari, M., \& Copin, Y. 2003, MNRAS, 342, 345

Cappellari, M., \& Emsellem, E. 2004, PASP, 116, 138

Colina, L., Piqueras López, J., Arribas, S., et al. 2015, A\&A, 578, A48

Collison, P. M., Saikia, D. J., Pedlar, A., Axon, D. J., \& Unger, S. W. 1994, MNRAS, 268, 203

Combes, F., García-Burillo, S., Boone, F., et al. 2004, A\&A, 414, 857

Combes, F., García-Burillo, S., Casasola, V., et al. 2013, A\&A, 558, A124

Combes, F., García-Burillo, S., Casasola, V., et al. 2014, A\&A, 565, A97

Comerón, S., Knapen, J. H., \& Beckman, J. E. 2008, A\&A, 485, 695

Comerón, S., Knapen, J. H., Beckman, J. E., et al. 2010, MNRAS, 402, 2462

Dahlem, M., Aalto, S., Klein, U., et al. 1990, A\&A, 240, 237

Dale, D. A., Sheth, K., Helou, G., Regan, M. W., \& Hüttemeister, S. 2005, AJ, 129, 2197

Davies, R. I., Maciejewski, W., Hicks, E. K. S., et al. 2014a, ApJ, 792, 101

Davies, R. L., Kewley, L. J., Ho, I.-T., \& Dopita, M. A. 2014b, MNRAS, 444, 3961

Davies, R. I., Sternberg, A., Lehnert, M., \& Tacconi-Garman, L. E. 2003, ApJ, 597, 907

Davies, R. I., Sternberg, A., Lehnert, M. D., \& Tacconi-Garman, L. E. 2005, ApJ 633, 105

Davies, R. I., Thomas, J., Genzel, R., et al. 2006, ApJ, 646, 754

Davies, R. I., Müller Sánchez, F., Genzel, R., et al. 2007, ApJ, 671, 1388

de Vaucouleurs, G., de Vaucouleurs, A., Corwin, Jr., H. G., et al. 1991, Third Reference Catalogue of Bright Galaxies, Volume I: Explanations and references. Volume II: Data for galaxies between $0^{\mathrm{h}}$ and $12^{\mathrm{h}}$. Volume III: Data for galaxies between $12^{\mathrm{h}}$ and $24^{\mathrm{h}}$

Diniz, M. R., Riffel, R. A., Storchi-Bergmann, T., \& Winge, C. 2015, MNRAS, 453,1727

Dopita, M. A., Shastri, P., Davies, R., et al. 2015, ApJS, 217, 12

Dors, Jr., O. L., Storchi-Bergmann, T., Riffel, R. A., \& Schimdt, A. A. 2008, A\&A, 482, 59

Draine, B. T., \& Woods, D. T. 1990, ApJ, 363, 464

Eisenhauer, F., Abuter, R., Bickert, K., et al. 2003, Instrument Design and Performance for Optical/Infrared Ground-based Telescopes, eds. M. Iye, \& A. F. M. Moorwood, SPIE Conf. Ser., 4841, 1548

Elmegreen, B. G. 1994, ApJ, 425, L73

Emsellem, E., Greusard, D., Combes, F., et al. 2001, A\&A, 368, 52

Emsellem, E., Fathi, K., Wozniak, H., et al. 2006, MNRAS, 365, 367

Esquej, P., Alonso-Herrero, A., González-Martín, O., et al. 2014, ApJ, 780, 86

Falcón-Barroso, J., Bacon, R., Bureau, M., et al. 2006, MNRAS, 369, 529

Falcón-Barroso, J., Ramos Almeida, C., Böker, T., et al. 2014, MNRAS, 438, 329

Ferrarese, L., \& Merritt, D. 2000, ApJ, 539, L9

Fisher, D. B., \& Drory, N. 2016, Galactic Bulges, 418, 41

Forbes, D. A., Boisson, C., \& Ward, M. J. 1992, MNRAS, 259, 293

Galliano, E., \& Alloin, D. 2008, A\&A, 487, 519

Galliano, E., Alloin, D., Pantin, E., Lagage, P. O., \& Marco, O. 2005, A\&A, 438, 803

Galliano, E., Kissler-Patig, M., Tacconi-Garmann, L., \& Alloin, D. 2014, in Massive Young Star Clusters Near and Far: From the Milky Way to Reionization, 2013 Guillermo Haro Conf., 95

García-Burillo, S., \& Combes, F. 2012, J. Phys. Conf. Ser., 372, 012050

García-Burillo, S., Combes, F., Hunt, L. K., et al. 2003, A\&A, 407, 485

García-Burillo, S., Combes, F., Schinnerer, E., Boone, F., \& Hunt, L. K. 2005, A\&A, 441, 1011
García-Burillo, S., Combes, F., Usero, A., et al. 2014, A\&A, 567, A125 Garcia-Rissmann, A., Vega, L. R., Asari, N. V., et al. 2005, MNRAS, 359, 765 Gebhardt, K., Bender, R., Bower, G., et al. 2000, ApJ, 539, L13 Goodrich, R. W., Veilleux, S., \& Hill, G. J. 1994, ApJ, 422, 521 Graham, A. W. 2012, ApJ, 746, 113

Graham, A. W. 2016, Galactic Bulges, 418, 263

Graham, A. W., \& Driver, S. P. 2007, ApJ, 655, 77

Graham, A. W., \& Scott, N. 2013, ApJ, 764, 151

Gültekin, K., Richstone, D. O., Gebhardt, K., et al. 2009, ApJ, 698, 198 Häring, N., \& Rix, H.-W. 2004, ApJ, 604, L89

Hickox, R. C., Mullaney, J. R., Alexander, D. M., et al. 2014, ApJ, 782, 9

Hilz, M., Naab, T., \& Ostriker, J. P. 2013, MNRAS, 429, 2924

Ho, L. C., Li, Z.-Y., Barth, A. J., Seigar, M. S., \& Peng, C. Y. 2011, ApJS, 197, 21

Hollenbach, D., \& McKee, C. F. 1989, ApJ, 342, 306

Hopkins, P. F., \& Quataert, E. 2010, MNRAS, 407, 1529

Hopkins, P. F., Hernquist, L., Cox, T. J., \& Kereš, D. 2008, ApJS, 175, 356

Kauffmann, G., Heckman, T. M., Tremonti, C., et al. 2003, MNRAS, 346, 1055

Kennicutt, Jr., R. C. 1998a, ARA\&A, 36, 189

Kennicutt, Jr., R. C. 1998b, ApJ, 498, 541

Kewley, L. J., Dopita, M. A., Sutherland, R. S., Heisler, C. A., \& Trevena, J. 2001, ApJ, 556, 121

Koribalski, B., Dahlem, M., Mebold, U., \& Brinks, E. 1993, A\&A, 268, 14

Koribalski, B. S., Staveley-Smith, L., Kilborn, V. A., et al. 2004, AJ, 128, 16

Kormendy, J., \& Ho, L. C. 2013, ARA\&A, 51, 511

Kormendy, J., \& Richstone, D. 1995, ARA\&A, 33, 581

Kormendy, J., Bender, R., \& Cornell, M. E. 2011, Nature, 469, 374

Kotilainen, J. K., Forbes, D. A., Moorwood, A. F. M., van der Werf, P. P., \& Ward, M. J. 1996, A\&A, 313, 771

Krabbe, A., Sternberg, A., \& Genzel, R. 1994, ApJ, 425, 72

Krabbe, A., Böker, T., \& Maiolino, R. 2001, ApJ, 557, 626

Lançon, A., Hauschildt, P. H., Ladjal, D., \& Mouhcine, M. 2007, A\&A, 468, 205

Larkin, J. E., Armus, L., Knop, R. A., Soifer, B. T., \& Matthews, K. 1998, ApJS, 114,59

Läsker, R., Ferrarese, L., van de Ven, G., \& Shankar, F. 2014, ApJ, 780, 70

Leitherer, C., Schaerer, D., Goldader, J. D., et al. 1999, ApJS, 123, 3

Leitherer, C., Ortiz Otálvaro, P. A., Bresolin, F., et al. 2010, ApJS, 189, 309

Leitherer, C., Ekström, S., Meynet, G., et al. 2014, ApJS, 212, 14

Levesque, E. M., \& Leitherer, C. 2013, ApJ, 779, 170

Maciejewski, W., Teuben, P. J., Sparke, L. S., \& Stone, J. M. 2002, MNRAS, 329,502

Makarov, D., Prugniel, P., Terekhova, N., Courtois, H., \& Vauglin, I. 2014, A\&A, 570, A13

Maloney, P. R., Hollenbach, D. J., \& Tielens, A. G. G. M. 1996, ApJ, 466, 561

Marconi, A., \& Hunt, L. K. 2003, ApJ, 589, L21

Markwardt, C. B. 2009, in Astronomical Data Analysis Software and Systems XVIII, eds. D. A. Bohlender, D. Durand, \& P. Dowler, ASP Conf. Ser., 411, 251

Mazzalay, X., Saglia, R. P., Erwin, P., et al. 2013, MNRAS, 428, 2389

Mazzalay, X., Maciejewski, W., Erwin, P., et al. 2014, MNRAS, 438, 2036

Mazzuca, L. M., Knapen, J. H., Veilleux, S., \& Regan, M. W. 2008, ApJS, 174, 337

Moré, J. 1978, in Lect. Notes in Mathematics, Numerical Analysis, ed. G. Watson (Berlin: Heidelberg Springer), 630, 105

Morgan, W. W. 1958, PASP, 70, 364

Moser, L., Krips, M., Busch, G., et al. 2016, A\&A, 587, A137

Moser, L., Zuther, J., Busch, G., Valencia-S., M., \& Eckart, A. 2012, PoS (Seyfert 2012)069

Mouri, H. 1994, ApJ, 427, 777

Mouri, H., Kawara, K., \& Taniguchi, Y. 1993, ApJ, 406, 52

Müller Sánchez, F., Davies, R. I., Eisenhauer, F., et al. 2006, A\&A, 454, 481

Müller-Sánchez, F., Prieto, M. A., Hicks, E. K. S., et al. 2011, ApJ, 739, 69

Osterbrock, D. E., \& Ferland, G. J. 2006, Astrophysics of gaseous nebulae and active galactic nuclei, eds. D. E. Osterbrock, \& G. J. Ferland (Sausalito, CA: University Science Books)

Panuzzo, P., Bressan, A., Granato, G. L., Silva, L., \& Danese, L. 2003, A\&A, 409, 99

Pastoriza, M. G. 1975, Ap\&SS, 33, 173

Phillips, A. C. 1993, AJ, 105, 486

Riffel, R. A., \& Storchi-Bergmann, T. 2011, MNRAS, 411, 469

Riffel, R. A., Storchi-Bergmann, T., Winge, C., et al. 2008, MNRAS, 385, 1129

Riffel, R. A., Storchi-Bergmann, T., Dors, O. L., \& Winge, C. 2009, MNRAS, 393, 783

Riffel, R. A., Storchi-Bergmann, T., \& Nagar, N. M. 2010, MNRAS, 404, 166

Riffel, R., Riffel, R. A., Ferrari, F., \& Storchi-Bergmann, T. 2011, MNRAS, 416, 493

Riffel, R., Rodríguez-Ardila, A., Aleman, I., et al. 2013, MNRAS, 430, 2002 
Riffel, R. A., Vale, T. B., Storchi-Bergmann, T., \& McGregor, P. J. 2014, MNRAS, 442, 656

Riffel, R. A., Ho, L. C., Mason, R., et al. 2015a, MNRAS, 446, 2823

Riffel, R. A., Storchi-Bergmann, T., \& Riffel, R. 2015b, MNRAS, 451, 3587

Rodríguez-Ardila, A., Pastoriza, M. G., Viegas, S., Sigut, T. A. A., \& Pradhan, A. K. 2004, A\&A, 425, 457

Rodríguez-Ardila, A., Riffel, R., \& Pastoriza, M. G. 2005, MNRAS, 364, 1041

Rosenberg, M. J. F., van der Werf, P. P., \& Israel, F. P. 2012, A\&A, 540, A116

Salak, D., Nakai, N., \& Kitamoto, S. 2014, PASJ, 66, 96

Salak, D., Nakai, N., Hatakeyama, T., \& Miyamoto, Y. 2016, ApJ, 823, 68

Sanders, D. B., Soifer, B. T., Elias, J. H., et al. 1988, ApJ, 325, 74

Savorgnan, G. A. D. 2016, ApJ, 821, 88

Savorgnan, G. A. D., Graham, A. W., Marconi, A., \& Sani, E. 2016, ApJ, 817, 21

Scharwächter, J., McGregor, P. J., Dopita, M. A., \& Beck, T. L. 2013, MNRAS, 429,2315

Schawinski, K., Thomas, D., Sarzi, M., et al. 2007, MNRAS, 382, 1415

Schawinski, K., Koss, M., Berney, S., \& Sartori, L. F. 2015, MNRAS, 451, 2517

Schmidt, M. 1959, ApJ, 129, 243

Scoville, N. Z., Hall, D. N. B., Ridgway, S. T., \& Kleinmann, S. G. 1982, ApJ, 253,136
Seo, W.-Y., \& Kim, W.-T. 2013, ApJ, 769, 100

Sérsic, J. L., \& Pastoriza, M. 1965, PASP, 77, 287

Sharp, R. G., \& Bland-Hawthorn, J. 2010, ApJ, 711, 818

Smajić, S., Fischer, S., Zuther, J., \& Eckart, A. 2012, A\&A, 544, A105

Smajić, S., Moser, L., Eckart, A., et al. 2014, A\&A, 567, A119

Smajić, S., Moser, L., Eckart, A., et al. 2015, A\&A, 583, A104

Sternberg, A., \& Dalgarno, A. 1989, ApJ, 338, 197

Storchi-Bergmann, T., Lopes, R. D. S., McGregor, P. J., et al. 2010, MNRAS, 402, 819

Tacconi-Garman, L. E., Sternberg, A., \& Eckart, A. 1996, AJ, 112, 918

Tully, R. B., Rizzi, L., Shaya, E. J., et al. 2009, AJ, 138, 323

Turner, J., Kirby-Docken, K., \& Dalgarno, A. 1977, ApJS, 35, 281

Väisänen, P., Barway, S., \& Randriamanakoto, Z. 2014, ApJ, 797, L16

Valencia-S., M., Zuther, J., Eckart, A., et al. 2012, A\&A, 544, A129

Vázquez, G. A., \& Leitherer, C. 2005, ApJ, 621, 695

Veron-Cetty, M.-P., \& Veron, P. 1985, A\&A, 145, 425

Vitale, M., Fuhrmann, L., García-Marín, M., et al. 2015, A\&A, 573, A93

Winge, C., Riffel, R. A., \& Storchi-Bergmann, T. 2009, ApJS, 185, 186

Wolniewicz, L., Simbotin, I., \& Dalgarno, A. 1998, ApJS, 115, 293

Wozniak, H., Combes, F., Emsellem, E., \& Friedli, D. 2003, A\&A, 409, 469

Zuther, J., Iserlohe, C., Pott, J.-U., et al. 2007, A\&A, 466, 45 